\title{
Polytopes and the Mean Value Property
}

\author{
K. Iwasaki \\ Department of Mathematical Sciences, University of Tokyo, \\ 3-8-1 Komaba, Meguro-ku, Tokyo 153, Japan \\ iwasaki@ms.u-tokyo.ac.jp
}

\begin{abstract}
Let $P$ be any (not necessarily convex nor connected) solid polytope in the $n$ dimensional Euclidean space $\mathbb{R}^{n}$, and let $P(k)$ be the $k$-skeleton of $P$. Let $\mathcal{H}_{P(k)}$ be the set of all continuous functions satisfying the mean value property with respect to $P(k)$. For any $k=0,1, \ldots, n$, we show that $\mathcal{H}_{P(k)}$ is a finite-dimensional linear space of polynomials. This settles an open problem posed by Friedman and Littman [37] in 1962. Moreover, we show that if $P$ admits ample symmetry, then $\mathcal{H}_{P(k)}$ is a finite-dimensional linear space of harmonic polynomials. Some interesting examples are also given.
\end{abstract}

\section{Introduction}

Let $\mu$ be a nonnegative Borel measure with compact support $K$ and total mass $\mu(K)=1$ in the $n$-dimensional Euclidean space $\mathbb{R}^{n}$, and let $\Omega$ be an open subset of $\mathbb{R}^{n}$. A $\mathbb{C}$-valued continuous function $f$ in $\Omega$ is said to satisfy the $\mu$-mean value property if

$$
f(x)=\int_{K} f(x+r y) d \mu(y)
$$

holds for any $x \in \Omega$ and $0<r<r_{x}$, where $r_{x}$ is a sufficiently small positive constant depending on $x \in \Omega$ such that inf $f_{x \in C} r_{x}>0$ for any compact subset $C$ of $\Omega$. Let $\mathcal{H}_{\mu}(\Omega)$ be the set of all continuous functions in $\Omega$ satisfying the $\mu$-mean value property (1.1).

It is a classical problem to characterize the linear space $\mathcal{H}_{\mu}(\Omega)$. We briefly review its long history. In the case of two dimensions Walsh [75], Beckenbach and Reade [8], and Friedman [36] showed that if $\mu$ is the homogeneous distribution on the vertices, sides, or area of a regular convex $N$-gon, then $\mathcal{H}_{\mu}(\Omega)$ is precisely the harmonic polynomials of degree $\leq N$ having zero $N$ th derivatives in each of the directions of the radii of the polygons. See also [60] and [52]. In the case of three dimensions Beckenbach and Reade [9] gave a similar characterization of $\mathcal{H}_{\mu}(\Omega)$ when $\mu$ is the homogeneous distribution on the vertices of the regular convex solids. However, their work was incomplete for the dodecahedron and icosahedron. This gap was filled by Flatto [28]. He also gave a 
complete characterization of $\mathcal{H}_{\mu}(\Omega)$ when $\mu$ is the homogeneous distribution on the vertices, surface, or volume of an $n$-dimensional regular tetrahedron or octahedron. In these special cases, $\mathcal{H}_{\mu}(\Omega)$ becomes a finite-dimensional linear space.

Flatto [27] considered the problem in the general setting as formulated above and showed that $f \in \mathcal{H}_{\mu}(\Omega)$ if and only if $f$ is a weak solution of a certain infinite system of linear partial differential equations. He also pointed out that $\mathcal{H}_{\mu}(\Omega)$ is finite dimensional if and only if the characteristic variety of the system consists of the singleton set $\{0\}$. This was still previously noticed by Aronszajn [6] in a different context.

Then Friedman and Littman [37] discovered another system of partial differential equations characterizing the $\mu$-mean value property (1.1), which is more convenient in many respects. In terms of this system Friedman and Littman [37] and then Flatto [28] obtained sufficient conditions on $\mu$ under which $\mathcal{H}_{\mu}(\Omega)$ is finite dimensional. Unfortunately, except for very restricted cases, their conditions seem to be difficult to verify even for Euclidean measures of familiar figures. In fact, in the end of their paper Friedman and Littman [37] wrote: "The problem of proving the finite dimensionality of the space of functions satisfying the mean value property (1.1) for such simple bodies as convex polyhedra, ellipsoids, etc., with the ordinary Euclidean measure does not seem to be easy. ... (one sentence omitted)... . Both methods, however, lead to rather involved technical problems in trying to apply them even to very simple bodies."

Several authors gave partial answers to the finite-dimensionality problem. For example, Garsia and Rodemich [42] considered the case of product measures and, in particular, established the finite dimensionality for the homogeneous distribution on an $n$-dimensional parallelepipedon. The case of discrete measures was investigated in great detail. In this case, the finite dimensionality was established by Friedman and Littman [37] and Garsia [41] in a general setting. Then more detailed investigations were made concerning various properties of the space $\mathcal{H}_{\mu}(\Omega)$, e.g., $\operatorname{dim} \mathcal{H}_{\mu}(\Omega)$, deg $\mathcal{H}_{\mu}(\Omega)$, etc., where $\operatorname{deg} \mathcal{H}_{\mu}(\Omega)$ is the highest degree of the polynomials in $\mathcal{H}_{\mu}(\Omega)$. See [29], [32], and [33]. In another direction many authors considered the problem from the viewpoint of functional equations. See [2], [15], [10], [51], [53]-[58], [65], [68], [72], [77], etc. There is a vast literature on the related topics (see the References).

The finite-dimensionality problem for arbitrary polytopes seems to have been open since Friedman and Littman [37] posed the question. (The author searched the Mathematical Reviews (1940-1995) for all papers in which the words mean value property appear at least once.) The purpose of this paper is to settle this problem affirmatively.

In this paper a polytope in $\mathbb{R}^{n}$ means a finite union of closed convex solid polytopes in $\mathbb{R}^{n}$. So a polytope is not necessarily convex nor connected. Given a polytope $P$ in $\mathbb{R}^{n}$ and $k=0,1, \ldots, n$, let $P(k)$ be the $k$-skeleton of $P$, i.e., the union of all faces of $P$ of dimension $\leq k$. Let $v_{k}$ be the $k$-dimensional Euclidean measure on $P(k)$, $|P(k)|=v_{k}(P(k))$, the $k$-dimensional Euclidean volume of $P(k)$. Then our problem is to describe those continuous functions $f$ in $\Omega$ which satisfy the $\mu$-mean value property (1.1) with respect to the measure $\mu=v_{k} /|P(k)|$. In this special case, the condition (1.1) is rewritten as

$$
f(x)=\frac{1}{|P(k)|} \int_{P(k)} f(x+r y) d v_{k}(y) \quad\left(0<r<r_{x}\right) .
$$

This condition is referred to as the $P(k)$-mean value property. Let $\mathcal{H}_{P(k)}(\Omega)$ be the set 
of all continuous functions in $\Omega$ satisfying (1.2). The problem of describing $\mathcal{H}_{P(k)}(\Omega)$ is referred to as the $k$-skeleton problem. For $k=0, n-1$, and $n$, the $k$-skeleton problem is also referred to as the vertex, surface, and volume problem, respectively (see [28]). Previously only these three problems (for only special figures) have been considered.

Let $x=\left(x_{1}, \ldots, x_{n}\right)$ denote a standard coordinate system of $\mathbb{R}^{n}$. We set $\partial=$ $\left(\partial_{1}, \ldots, \partial_{n}\right)$, where $\partial_{j}=\partial / \partial x_{j}$. Let $\mathbb{C}[\partial]=\mathbb{C}\left[\partial_{1}, \ldots, \partial_{n}\right]$ be the ring of partial differential operators with constant coefficients. The most fundamental result in this paper is the following:

Theorem 1.1. Let $P$ be any (not necessarily convex nor connected) polytope in $\mathbb{R}^{n}$, and let $\Omega$ be any open subset of $\mathbb{R}^{n}$. Then for each $k=0,1, \ldots, n$, the following assertions hold:

(1) $\mathcal{H}_{P(k)}(\Omega)$ is a finite-dimensional linear space of polynomials.

(2) A basis of $\mathcal{H}_{P(k)}(\Omega)$ can be taken from homogeneous polynomials.

(3) $\mathcal{H}_{P(k)}(\Omega)$ admits a structure of $\mathbb{C}[\partial]$-module.

For $k=0$ the result is not new since the vertex problem is a special case of the discrete measure problem for which the finite dimensionality is well known as mentioned previously. Theorem 1.1 is established in Section 4.

Remark 1.2. It can be shown that the restriction map $\mathcal{H}_{P(k)}\left(\mathbb{R}^{n}\right) \rightarrow \mathcal{H}_{P(k)}(\Omega)$ is an isomorphism (see Proposition 3.9). So $\mathcal{H}_{P(k)}(\Omega)$ is independent of the domain $\Omega$. This remark allows us to simplify the notation as $\mathcal{H}_{P(k)}=\mathcal{H}_{P(k)}(\Omega)$. Sometimes we use this simplified notation.

\section{Main Results}

We state the main results of this paper other than Theorem 1.1. They are either a main step for establishing Theorem 1.1 or an amplification of Theorem 1.1. First we introduce some notations: $x=\left(x_{1}, \ldots, x_{n}\right)$ and $y=\left(y_{1}, \ldots, y_{n}\right)$ are vectors in $\mathbb{R}^{n}$ while $\xi=$ $\left(\xi_{1}, \ldots, \xi_{n}\right)$ and $\eta=\left(\eta_{1}, \ldots, \eta_{n}\right)$ are vectors in $\mathbb{C}^{n}$. We set $\langle\xi, \eta\rangle=\xi_{1} \eta_{1}+\cdots+\xi_{n} \eta_{n}$. Let $\left\{P_{i_{j}}\right\}_{i_{j} \in I j}$ be the set of $j$-dimensional faces of $P, H_{i_{j}}$ the $j$-dimensional affine subspace of $\mathbb{R}^{n}$ containing $P_{i_{j}}, \pi_{i_{j}}: \mathbb{R}^{n} \rightarrow H_{i_{j}}$ the orthogonal projection from $\mathbb{R}^{n}$ down to the subspace $H_{i_{j}}$. Let $p_{i_{j}} \in \mathbb{R}^{n}$ be the vectors defined by

$$
p_{i_{j}}=\pi_{i_{j}}(0) \in H_{i_{j}} .
$$

We remark that $H_{i_{n}}=\mathbb{R}^{n}$ and $p_{i_{n}}=0$ for any $i_{n} \in I_{n}$. For $i_{j} \in I_{j}$ and $i_{j+1} \in I_{j+1}$ we write $i_{j} \prec i_{j+1}$ if $P_{i_{j}}$ is a face of $P_{i_{j+1}}$. For $i_{j} \prec i_{j+1}$ let $\mathbf{n}_{i_{j} i_{j+1}}$ be the outer unit normal vector of $\partial P_{i_{j+1}}$ in $H_{i_{j+1}}$ at the face $P_{i_{j}}$. The vector $p_{i_{j}}-p_{i_{j+1}}$ is parallel to $\mathbf{n}_{i_{j} i_{j+1}}$, so that we can define $\left[i_{j}: i_{j+1}\right] \in \mathbb{R}$ by the relation

$$
p_{i_{j}}-p_{i_{j+1}}=\left[i_{j}: i_{j+1}\right] \mathbf{n}_{i_{i} i_{j+1}} .
$$

Let $I(k)$ be the index set defined by

$$
I(k)=\left\{i=\left(i_{0}, i_{1}, \ldots, i_{k}\right) ; i_{j} \in I_{j}, i_{0} \prec i_{1} \prec \cdots \prec i_{k}\right\} .
$$


For $i=\left(i_{0}, i_{1}, \ldots, i_{k}\right) \in I(k)$ we set

$$
[i]= \begin{cases}1 & (k=0), \\ {\left[i_{0}: i_{1}\right]\left[i_{1}: i_{2}\right] \cdots\left[i_{k-1}: i_{k}\right]} & (k=1,2, \ldots, n) .\end{cases}
$$

Let $h_{m}^{(j)}(\xi)$ be the complete symmetric polynomial of degree $m$ in $j$-variables:

$$
h_{m}^{(j)}\left(\xi_{1}, \ldots, \xi_{j}\right)=\sum_{m_{1}+\cdots+m_{j}=m} \xi_{1}^{m_{1}} \xi_{2}^{m_{2}} \ldots \xi_{j}^{m_{j}}
$$

where the summation is taken over all $j$-tuples $\left(m_{1}, \ldots, m_{j}\right)$ of nonnegative integers satisfying the indicated condition (see [64]).

The first theorem gives a characterization of the $P(k)$-mean value property (1.2) in terms of a system of partial differential equations.

Theorem 2.1. Any $f \in \mathcal{H}_{P(k)}(\Omega)$ is real-analytic and satisfies the system of partial differential equations:

$$
\tau_{m}^{(k)}(\partial) f=0 \quad(m=1,2,3, \ldots),
$$

where $\tau_{m}^{(k)}(\xi)$ is the homogeneous polynomial of degree $m$ defined by

$$
\tau_{m}^{(k)}(\xi)=\sum_{i \in I(k)}[i] h_{m}^{(k+1)}\left(\left\langle p_{i_{0}}, \xi\right\rangle,\left\langle p_{i_{1}}, \xi\right\rangle, \ldots,\left\langle p_{i_{k}}, \xi\right\rangle\right) .
$$

Conversely, any weak solution of (2.6) is real-analytic and belongs to $\mathcal{H}_{P(k)}(\Omega)$.

Theorems 2.1 and 2.3 below play a key role in establishing Theorem 1.1. Theorem 2.1 is also used to establish a relation between the volume problem and the surface problem.

Theorem 2.2. For each $i_{n-1} \in I_{n-1}$ there exists a unique $i_{n} \in I_{n}$ such that $i_{n-1} \prec i_{n}$. We set $c_{i_{n-1}}=\left[i_{n-1}: i_{n}\right]$. Assume that $c_{i_{n-1}}$ is independent of $i_{n-1} \in I_{n-1}$. Then,

$$
\mathcal{H}_{P(n-1)}(\Omega)=\mathcal{H}_{P(n)}(\Omega) \text {. }
$$

Friedman and Littman [37] have already obtained a system of partial differntial equations which characterizes the $\mu$-mean value property (1.1) for a general measure $\mu$ (see (3.1) in Theorem 3.1). In fact, our system (2.6) turns out to be equivalent to their system (3.1) for the special measure $\mu=v_{k} /|P(k)|$ (see Theorem 3.3). The important point here is that their system admits such an explicit representation as (2.6)-(2.7). They gave no such explicit representation. Theorems 2.1 and 2.2 are established in Section 3.

We proceed to the finite-dimensionality problem: $I s \mathcal{H}_{P(k)}(\Omega)$ finite dimensional?

The following theorem plays a crucial role in solving this problem.

Theorem 2.3. The system of algebraic equations:

$$
\tau_{m}^{(k)}(\xi)=0 \quad(m=1,2,3, \ldots),
$$

has $\xi=0$ as its only common root in $\mathbb{C}^{n}$. 
Theorem 2.3 and a standard technique used by Flatto [27], [28] and Friedman and Littman [37] (a method based on the Hilbert Nullstellensatz) lead to an affirmative answer to the finite-dimensionality problem. (see Theorem 3.2 ). Theorem 2.3 is established in Section 4.

Another interesting problem is: When is any function in $\mathcal{H}_{P(k)}(\Omega)$ harmonic? This problem is closely related to the symmetry of $P(k)$.

Theorem 2.4. Let $G \subset O(n)$ be a group of symmetries of $P(k)$. Assume that, for any nonzero vector $v \in \mathbb{R}^{n}$, the vectors belonging to the orbit $G v$ span the entire Euclidean space $\mathbb{R}^{n}$. Then $\mathcal{H}_{P(k)}(\Omega)$ is a finite-dimensional linear space of harmonic polynomials.

We remark that Theorem 2.4 applies to each skeleton $P(k)$ of any (convex or nonconvex) regular polytopes $P$ in $\mathbb{R}^{n}$ with center at the origin, $G=G_{P}$ being the complete symmetry group of $P$. Theorem 2.4 is established in Section 5 .

Following Remark 1.2 we use the simplified notation $\mathcal{H}_{P(k)}=\mathcal{H}_{P(k)}(\Omega)$. Theorem 1.1(2) asserts that there exists a direct sum decomposition:

$$
\mathcal{H}_{P(k)}=\bigoplus_{m \geq 0}^{\text {finite }} \mathcal{H}_{P(k)}(m),
$$

where $\mathcal{H}_{P(k)}(m)$ is the linear space of all homogeneous polynomials of degree $m$ satisfying the $P(k)$-mean value property (1.2). Moreover Theorem 1.1(3) asserts that $\mathcal{H}_{P(k)}$ carries a structure of $\mathbb{C}[\partial]$-module. Several problems naturally arise. For examples the following problems are interesting from a combinatorial point of view.

\section{Problem 2.5.}

(1) Determine $\operatorname{dim} \mathcal{H}_{P(k)}$ and construct a basis of $\mathcal{H}_{P(k)}$.

(2) Determine $\operatorname{dim} \mathcal{H}_{P(k)}(m)$ and construct a basis of $\mathcal{H}_{P(k)}(m)$.

(3) Determine the structure of $\mathcal{H}_{P(k)}$ as a $\mathbb{C}[\partial]$-module.

These problems are of particular interest when $P(k)$ admits ample symmetry. We consider the two-dimensional cases to which Theorem 2.4 applies effectively. Let $P$ be a polygon. The $k$-skeleton $P(k)(k=0,1,2)$ is said to admit a $\mathbb{Z}_{N}$-symmetry if it admits a cyclic group of rotations of order $N$ as a symmetry group. We identify $\mathbb{R}^{2}$ with the Gauss plane $\mathbb{C}$ with coordinate $z=x+\sqrt{-1} y$.

Theorem 2.6. Let $P$ be a polygon and assume that $P(k)$ admit a $\mathbb{Z}_{N}$-symmetry for an integer $N \geq 3$. Let $a_{P(k)} \in \mathbb{C}$ be the constant defined by

$$
a_{P(k)}=\int_{F} \bar{z}^{N} d v_{k}(z)
$$

where $F$ is a fundamental domain of $P(k)$ with respect to the $\mathbb{Z}_{N}$-action. Assume that 
the constant $a_{P(k)}$ does not vanish. Then $\mathcal{H}_{P(k)}$ is of dimension $2 N$ and

$$
\mathcal{H}_{P(k)}(m)= \begin{cases}\mathbb{C} & (m=0), \\ \mathbb{C} z^{m} \oplus \mathbb{C} z^{m} & (1 \leq m \leq N-1), \\ \mathbb{C} \operatorname{Im}\left(a_{P(k)} z^{N}\right) & (m=N), \\ \{0\} & (m \geq N+1) .\end{cases}
$$

As a $\mathbb{C}[\partial]$-module. $\mathcal{H}_{P(k)}$ is generated by the single element $\operatorname{Im}\left(a_{P(k)} z^{N}\right)$.

Theorem 2.6 is established in Section 5. This theorem applies to any regular starpolygon $\{N / M\}$ with center at the origin. See p. 93 of [20] for the definition of regular star-polygons and the notation $\{N / M\}$. In case $M=1,\{N\}$ is a regular convex $N$-gon.

Theorem 2.7. Let $P$ be a regular star-polygon $\{N / M\}$ with center at the origin, where $N$ and $M$ are coprime natural numbers such that $N>2 M$. Then for each $k=0,1,2$, $\mathcal{H}_{P(k)}$ is of dimension $2 N$. Let $(x, y)$ be an orthonormal coordinate system of $\mathbb{R}^{2}$ such that $P$ is symmetric with respect to the $x$-axis. Then $\mathcal{H}_{P(k)}(m)$ is given by (2.12) with $a_{P(k)}$ replaced by 1 . As $a \mathbb{C}[\partial]$-module $\mathcal{H}_{P(k)}$ is generated by the single element $\operatorname{Im}\left(z^{N}\right)$.

Theorem 2.7 is established in Section 6. Another application of Theorem 2.6 is also given in Section 6 (see Theorem 6.1).

For any finite subgroup $G$ of $G L(n, \mathbb{R})$ there exists the notion of $G$-harmonic functions due to Steinberg [74]. Let $\mathcal{H}_{G}$ be the linear space of all $G$-harmonic functions. Steinberg [74] established the inequality:

$$
\operatorname{dim} \mathcal{H}_{G} \geq|G| .
$$

We consider the $G_{P(k)}$-harmonic functions, where $G_{P(k)} \subset O(n)$ is the complete symmetry group of $P(k)$. For any $m=1,2,3, \ldots, \tau_{m}^{(k)}(\xi)$ is a $G_{P(k)}$-invariant polynomial. Hence Theorem 2.1 leads to the inclusion:

$$
\mathcal{H}_{G_{P(k)}} \subset \mathcal{H}_{P(k)}
$$

So (2.13) and (2.14) yield a lower bound for the dimension of $\mathcal{H}_{P(k)}$ :

$$
\operatorname{dim} \mathcal{H}_{P(k)} \geq\left|G_{P(k)}\right| \text {. }
$$

Steinberg [74] showed that if $G$ is a finite reflection group, then the inequality in (2.13) is just an equality and as a $\mathbb{C}[\partial]$-module $\mathcal{H}_{G}$ is generated by $\Delta_{G}$, where $\Delta_{G}$ is the fundamental alternating polynomial for $G$. We notice that the dimension of $\mathcal{H}_{P(k)}$ in Theorem 2.7 coincides with the order of the dihedral group $\mathbf{D}_{2 N}$ (a finite reflection group) to which the complete symmetry group of the regular star-polygon $\{N / M\}$ is isomorphic. Theorem 2.7 asserts that the inclusion (2.14) is, in fact, an equality for any regular star-polygon $\{N / M\}$. This observation leads to the following:

Problem 2.8. Find (necessary and) sufficient conditions for the equality

$$
\mathcal{H}_{G_{P(k)}}=\mathcal{H}_{P(k)}
$$


For any regular convex polyhedra $P$ in $\mathbb{R}^{3}$ with center at the origin we have (2.16) for every $k=0,1,2,3$ (see [59]). Flatto [28], and Flatto and Wiener [35] established (2.16) for the regular tetrahedron and octahedron in $\mathbb{R}^{n}$ (for $k=0, n-1, n$ ), for the dodecahedron and icosahedron in $\mathbb{R}^{3}$, and for all regular convex solids in $\mathbb{R}^{4}$ (for $k=0$ ). See also [47]. Even in dimension 1 there exists an example for which the inclusion (2.14) is strict (see (6.10) and (6.11)). Also in dimension 2 if a polygon $P$ satisfies the assumption of Theorem 2.6 and has $\mathbb{Z}_{N}$ as the complete symmetry group, then the inclusion (2.14) is strict. There are uncountably many such polygons.

We conclude this section by giving two variations of our problem.

Problem 2.9. In the definition of the $P(k)$-mean value property we assumed (1.2) for all radii $r$ smaller than $r_{x}$. What happens if we assume (1.2) for just one radius, or two or a fixed finite number? This problem is clearly related to the Pompeiu problem in integral geometry (see [11]-[13] and [79]).

Problem 2.10. Our problem makes sense for "polytopes" on any complete Riemannian manifold of constant curvature, and even more generally on symmetric spaces. What happens in such general cases?

Problems 2.9 and 2.10 were suggested by one of the referees.

\section{Differential Equations}

Flatto [27] first discovered an infinite system of partial differential equations which characterizes the $\mu$-mean value property (1.1). Then Friedman and Littman [37] discovered another system, which is more convenient in many respects. See also [78]. Let us recall some of their results needed in the sequel. We use the usual multi-index notation: for $\alpha=\left(\alpha_{1}, \ldots, \alpha_{n}\right)$ we set $x^{\alpha}=x_{1}^{\alpha_{1}} \cdots x_{n}^{\alpha_{n}}, \partial^{\alpha}=\partial_{1}^{\alpha_{1}} \cdots \partial_{n}^{\alpha_{n}},|\alpha|=\alpha_{1}+\cdots+\alpha_{n}$, and $\alpha !=\alpha_{1} ! \alpha_{2} ! \cdots \alpha_{n} !$. The following theorems are fundamental in studying the $\mu$-mean value property (1.1).

Theorem 3.1 [37, Theorem 1]. Let $\mu$ be a nonnegative Borel measure in $\mathbb{R}^{n}$ with compact support $K$ and total mass $\mu(K)=1$. Assume that $K$ is not contained in any $(n-1)$-dimensional hyperplane. Then any $f \in \mathcal{H}_{\mu}(\Omega)$ is real-analytic and satisfies the infinite system of partial differential equations:

$$
\sigma_{m}(\partial) f=0 \quad(m=1,2,3, \ldots)
$$

where $\sigma_{m}(\xi)$ is the homogeneous polynomial of degree $m$ defined by

$$
\sigma_{m}(\xi)=\sum_{|\alpha|=m} \frac{m !}{\alpha !} s_{\alpha} \xi^{\alpha}
$$


where the coefficient $s_{\alpha}$ is the $\alpha$-moment of the measure $\mu$ :

$$
s_{\alpha}=\int_{K} y^{\alpha} d \mu(y)
$$

Conversely, any weak solution $f$ of $(3.1)$ in $\Omega$ is real-analytic and belongs to $\mathcal{H}_{\mu}(\Omega)$.

Our multi-index notation is slightly different from that in [37]. So (3.1)-(3.3) are slightly different from (1.2)-(1.3) in [37], but equivalent to them.

Theorem 3.2 [37, Theorem 2]. The linear space $\mathcal{H}_{\mu}(\Omega)$ is finite dimensional if and only if the system of algebraic equations:

$$
\sigma_{m}(\xi)=0 \quad(m=1,2,3, \ldots),
$$

has $\xi=0$ as its only common root in $\mathbb{C}^{n}$. If this is the case then any $f \in \mathcal{H}_{\mu}(\Omega)$ is a polynomial.

We would like to find an explicit representation of the polynomials $\sigma_{m}(\xi)$ in our situation. The following theorem gives an answer to this problem.

Theorem 3.3. For the measure $\mu=v_{k} /|P(k)|$ the polynomials $\sigma_{m}(\xi)$ in (3.2) are represented as

$$
\sigma_{m}(\xi)=\frac{\tau_{m}^{(k)}(\xi)}{|P(k)| \prod_{j=1}^{k}(m+j)} \quad(m=1,2,3, \ldots)
$$

where $\tau_{m}^{(k)}(\xi)$ are the polynomials defined by (2.7).

In our situation the coefficients $s_{\alpha}$ of $\sigma_{m}(\xi)$, and hence the polynomials $\sigma_{m}(\xi)$ themselves, may be computed rather explicitly by the repeated use of integration by parts or the Stokes theorem. But so far we have not succeeded along this line. A difficulty lies in the fact that the crucial role played by the vectors $p_{i}$ is far from clear from this point of view. So we take another approach to derive the formula (3.5). Our approach is based on the successive applications of the radial differential operator $\delta_{r}=r \partial_{r}$ to the $P(k)$-mean value property (1.2).

In addition to those in Section 2 we use the following notations. For $t=0,1, \ldots, k+1$, let $I(t, k)$ be the index set defined by

$$
I(t, k)= \begin{cases}\left\{i=\left(i_{t}, i_{t+1}, \ldots, i_{k}\right) ; i_{j} \in I_{j}, i_{t} \prec i_{t+1} \prec \cdots \prec i_{k}\right\} & (t=0, \ldots, k), \\ \{\emptyset\} & (t=k+1) .\end{cases}
$$

We remark that $I(0, k)=I(k)$, where $I(k)$ is defined by (2.3). For $i=\left(i_{l}, \ldots, i_{k}\right) \in$ $I(t, k)$ we set

$$
[i]= \begin{cases}{\left[i_{t}: i_{t+1}\right]\left[i_{t+1}: i_{t+2}\right] \cdots\left[i_{k-1}: i_{k}\right]} & (t=0, \ldots, k-1), \\ 1 & (t=k) .\end{cases}
$$


Let $D_{i_{j}}$ be the first-order partial differential operator defined by

$$
\left(D_{i_{j}} f\right)(x)=\left\langle p_{i_{j}}, \partial\right\rangle f(x)=\lim _{\varepsilon \rightarrow 0} \frac{1}{\varepsilon}\left\{f\left(x+\varepsilon p_{i_{j}}\right)-f(x)\right\} .
$$

Let $v_{i_{j}}$ be the Euclidean measure on the affine subspace $H_{i j}$. It also denotes the Euclidean measure on $x+r H_{i_{j}}$ for every $x \in \mathbb{R}^{n}$ and $r>0$. We remark that for $i_{0} \in I_{0}, H_{i_{0}}$ is the singleton set $\left\{p_{i_{0}}\right\}$ and $v_{i_{0}}$ is the delta measure at the point $p_{i_{0}}$. We recall that $v_{k}$ is the Euclidean measure on $P(k)$. It also denotes the Euclidean measure on $x+r P(k)$. We remark that $v_{k}=v_{i_{k}}$ on $x+r P_{i_{k}}$ for $i_{k} \in I_{k}$.

Lemma 3.4. Let $f$ be a $C^{1}$-function in $\mathbb{R}^{n}$. Then for each $j=1,2, \ldots, n$ and $i_{j} \in I_{j}$ we have

$$
\begin{array}{rl}
\delta_{r} \int_{x+r P_{i_{j}}} f & d v_{i_{j}} \\
& =r \int_{x+r P_{i_{j}}} D_{i_{j}} f d v_{i_{j}}+r \sum_{i_{j-1}<i_{j}}\left[i_{j-1}: i_{j}\right] \int_{x+r P_{i_{j-1}}} f d v_{i_{j-1}} .
\end{array}
$$

Proof. For any fixed $x \in \mathbb{R}^{n}, r>0$, and $i_{j} \in I_{j}$, we set

$$
u(\varepsilon)=\int_{x+e^{\varepsilon} r P_{i_{j}}} f d v_{i_{j}} \quad(\varepsilon \in \mathbb{R}) .
$$

Let $\pi_{\varepsilon}: \mathbb{R}^{n} \rightarrow x+e^{\varepsilon} r H_{i_{j}}$ be the orthogonal projection from the Euclidean space $\mathbb{R}^{n}$ down to the affine subspace $x+e^{\varepsilon} r H_{i_{j}}$. We write $u(\varepsilon)=u_{1}(\varepsilon)+u_{2}(\varepsilon)$, where

$$
u_{1}(\varepsilon)=\int_{\pi_{\varepsilon}\left(x+r P_{i_{j}}\right)} f d v_{i_{j}}, \quad u_{2}(\varepsilon)=\int_{x+e^{\varepsilon} r P_{i_{j}}} f d v_{i_{j}}-\int_{\pi_{\varepsilon}\left(x+r P_{i_{j}}\right)} f d v_{i_{j}} .
$$

A geometric consideration based on Figs. 3.1 and 3.2 yields

$$
\begin{aligned}
& u_{1}(\varepsilon)=u(0)+\varepsilon r \int_{x+r P_{i_{j}}} D_{i_{j}} f d v_{i_{j}}+O\left(\varepsilon^{2}\right), \\
& u_{2}(\varepsilon)=\varepsilon r \sum_{i_{j-1}<i_{j}}\left[i_{j-1}: i_{j}\right] \int_{x+r P_{i_{j-1}}} f d v_{i_{j-1}}+O\left(\varepsilon^{2}\right) .
\end{aligned}
$$

We use the fact that the radial differential operator $\delta_{r}$ is the infinitesimal generator of the similarity transformations with base point at the origin. This implies that the left-hand side of (3.9) equals $(d u / d \varepsilon)_{\varepsilon=0}$. But (3.10) shows that $(d u / d \varepsilon)_{\varepsilon=0}$ equals the right-hand side of (3.9). The proof is complete.

For $i=\left(i_{t}, i_{t+1}, \ldots, i_{k}\right) \in I(t, k)$ let $A_{m}^{(i)}$ be the $m$ th-order homogeneous partial differential operator defined by

$$
A_{m}^{(i)}= \begin{cases}h_{m}^{(k-t+1)}\left(D_{i_{t}}, D_{i_{t+1}}, \ldots, D_{i_{k}}\right) & (t=0, \ldots, k), \\ 0 & (t=k+1)\end{cases}
$$




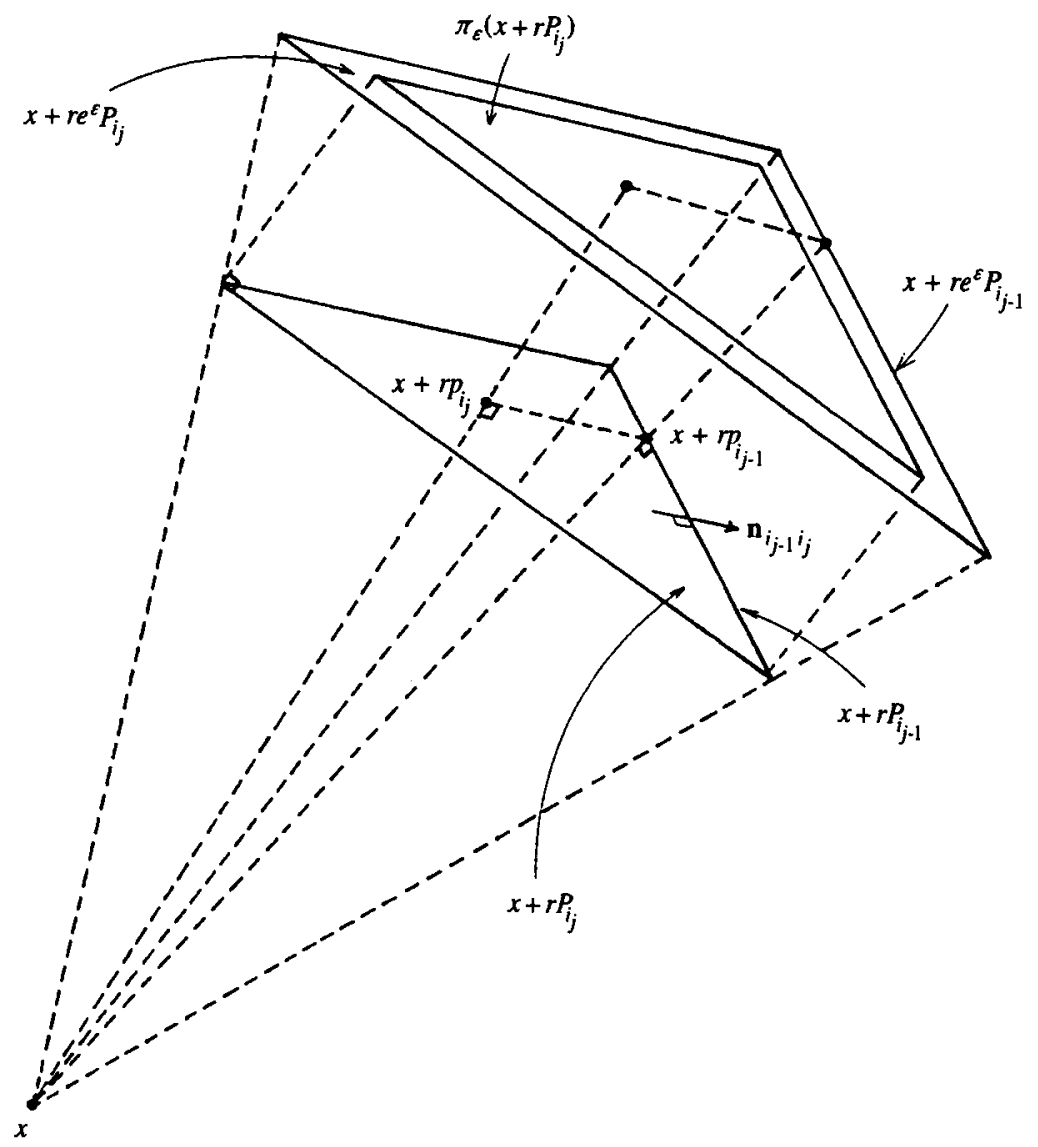

Fig. 3.1

where $h_{m}^{(j)}(\xi)$ is defined by (2.5). At first glance the right-hand side of (3.11) depends on $t$ and $k$ while the left-hand side does not. We remark that, however, the index $i$ on the left-hand side depends implicitly on $t$ and $k$. For $i=\left(i_{t}, i_{t+1}, \ldots, i_{k}\right) \in I(t, k)$ we define $i^{\prime} \in I(t+1, k)$ by

$$
i^{\prime}= \begin{cases}\left(i_{t+1}, \ldots, i_{k}\right) & (t=0, \ldots, k-1) \\ \emptyset & (t=k)\end{cases}
$$

The definition (2.5) of the complete symmetric polynomials $h_{m}^{(j)}(\xi)$ and the definition (3.11) of $A_{m}^{(i)}$ readily yield the following:

Lemma 3.5. For $i \in I(t, k)(t=0, \ldots, k)$ the following recurrence relation holds:

$$
A_{m}^{(i)}= \begin{cases}1 & (m=0) \\ D_{i_{1}} A_{m-1}^{(i)}+A_{m}^{\left(i^{\prime}\right)} & (m \geq 1) .\end{cases}
$$




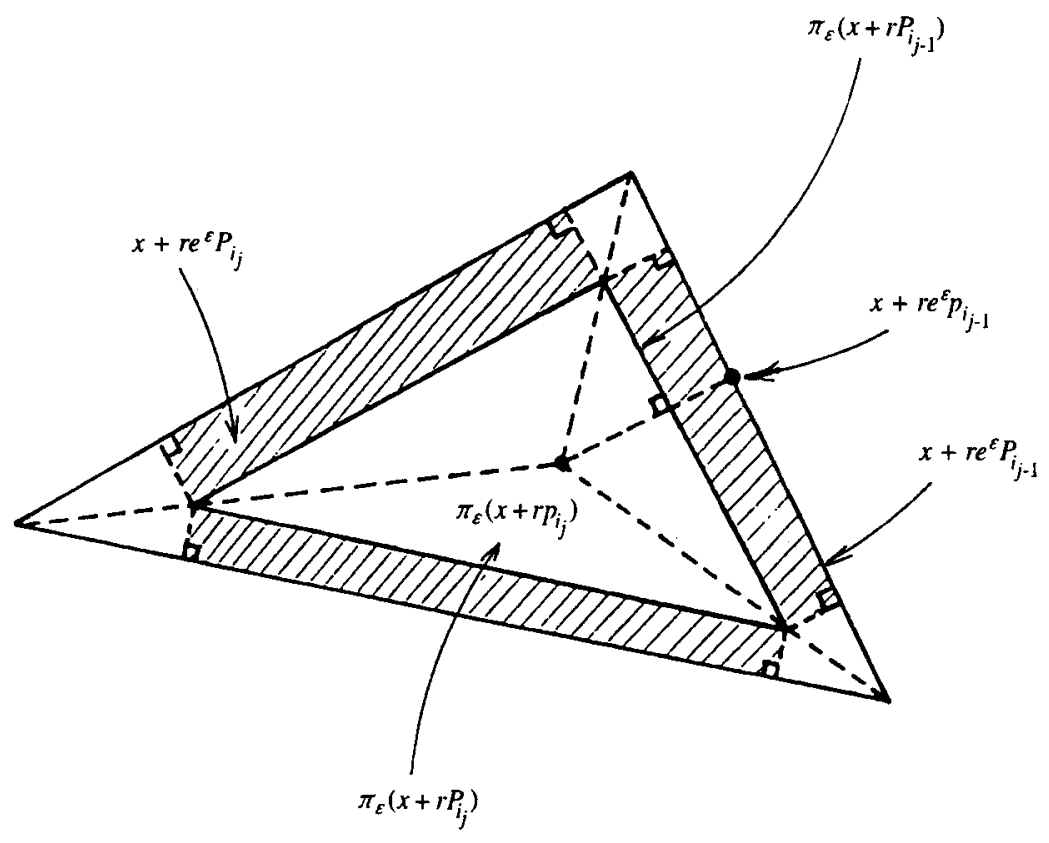

Fig. 3.2

Lemma 3.6. For any $C^{\infty}$-function $f$ let $F(x, r)$ be the function defined by

$$
F(x, r)=\int_{P(k)} f(x+r y) d v_{k}(y)=\frac{1}{r^{k}} \int_{x+r P(k)} f d v_{k},
$$

Then the following equality holds for $t=0,1, \ldots, k$ :

$$
r^{t} \prod_{j=t+1}^{k}\left(\delta_{r}+j\right) F(x, r)=\sum_{j=t}^{k} \sum_{i \in I(j, k)}[i] \int_{x+r P_{i j}} A_{j-t}^{(i)} f d v_{i_{j}} .
$$

Proof. The definition (3.14) of $F(x, r)$ is rewritten as

$$
r^{k} F(x, r)=\sum_{i_{k} \in I_{k}} \int_{x+r P_{i_{k}}} f d v_{i_{k}} .
$$

This is nothing but (3.15.k). The proof goes by downward induction on $t$. If (3.15.t) holds then an application of $\delta_{r}$ to $(3.15 . t)$ yields

$$
\begin{aligned}
r^{t} \prod_{j=t}^{n}\left(\delta_{r}+j\right) F(x, r)= & \sum_{j=t}^{k} \sum_{i \in I(j, k)}[i] \int_{x+r P_{i_{j}}} r D_{i_{j}} A_{j-t}^{(i)} f d v_{i_{j}} \\
& +\sum_{j=t}^{k} \sum_{i \in I(j, k)} \sum_{i_{j-1}<i_{j}}[i] r\left[i_{j-1}: i_{j}\right] \int_{x+r P_{i j-1}} A_{j-t}^{(i)} f d v_{i_{j-1}}
\end{aligned}
$$




$$
\begin{aligned}
= & r \sum_{j=t}^{m} \sum_{i \in I(j, k)}[i] \int_{x+r P_{i_{j}}} D_{i_{j}} A_{j-t}^{(i)} f d v_{i_{j}} \\
& +r \sum_{j=t}^{k} \sum_{i \in I(j-1, k)}[i] \int_{x+r P_{i_{j-1}}} A_{j-t}^{\left(i^{\prime}\right)} f d v_{i_{j-1}} \\
= & r \sum_{j=t}^{k} \sum_{i \in I(j, k)}[i] \int_{x+r P_{i_{j}}}\left\{D_{i_{j}} A_{j-t}^{(i)}+A_{j-t+1}^{\left(i^{\prime}\right)}\right\} f d v_{i_{j}} \\
& +r \sum_{i \in I(t-1, k)}[i] \int_{x+r P_{i_{t-1}}} A_{0}^{\left(i^{\prime}\right)} f d \nu_{i_{t-1}} \\
= & r \sum_{j=t-1}^{k} \sum_{i \in I(j, k)}[i] \int_{x+r P_{i_{j}}} A_{j-t+1}^{(i)} f d v_{i_{j}},
\end{aligned}
$$

where the first equality follows from (3.9), the second from (3.7), and the fourth from (3.13), respectively. Dividing these equalities by $r$ we establish $(3.15 . t-1)$. Hence the proof is complete.

We remark that $P_{i_{0}}=\left\{p_{i_{0}}\right\}$ is a singleton set for any $i_{0} \in I_{0}$. Hence (3.15.0) is rewritten as

$$
\begin{aligned}
\prod_{j=1}^{k}\left(\delta_{r}+j\right) F & (x, r) \\
= & \sum_{i \in I(k)}[i] f\left(x+r p_{i_{0}}\right)+\sum_{j=1}^{k} \sum_{i \in I(j, k)}[i] \int_{x+r P_{i_{j}}} A_{j}^{(i)} f d v_{i_{j}}
\end{aligned}
$$

Lemma 3.7. Let $F(x, r)$ be defined by (3.14). Then the following formula holds for every nonnegative integer $m$ :

$$
\begin{aligned}
& \prod_{j=1}^{k}\left(\delta_{r}+m+j\right) \partial_{r}^{m} F(x, r) \\
& \quad=\sum_{i \in I(k)}[i]\left(A_{m}^{(i)} f\right)\left(x+r p_{i_{0}}\right)+\sum_{j=1}^{k} \sum_{i \in I(j, k)}[i] \int_{x+r P_{i_{j}}} A_{m+j}^{(i)} f d \nu_{i_{j}} . \quad(3.17 . m)
\end{aligned}
$$

Proof. Since (3.17.0) is just the same as (3.16), (3.17.0) is already established in Lemma 3.6. The proof goes by induction. If $(3.17 . \mathrm{m})$ holds then an application of the radial differential operator $\delta_{r}$ to $(3.17 . \mathrm{m})$ yields

$$
\begin{aligned}
& r \prod_{k=j}^{k}\left(\delta_{r}+m+1+j\right) \partial_{r}^{m+1} F(x, r) \\
& \quad=r \sum_{i \in I(k)}[i]\left(D_{i_{0}} A_{m}^{(i)}\right)\left(x+r p_{i_{0}}\right)+\sum_{j=1}^{k} \sum_{i \in I(j, k)}[i] \int_{x+r P_{i_{j}}} r D_{i_{j}} A_{m+j}^{(i)} f d v_{i_{j}}
\end{aligned}
$$




$$
+\sum_{j=1}^{k} \sum_{i \in I(j, k)} \sum_{i_{j-1}<i_{j}}[i] r\left[i_{j-1}: i_{j}\right] \int_{x+r P_{i_{j-1}}} A_{m+j}^{(i)} f d v_{i_{j-1}},
$$

where Lemma 3.4 is used. Manipulating the second and third terms in the right-hand side in a similar manner as in the proof of Lemma 3.6, we obtain

$$
\begin{aligned}
& r \prod_{j=1}^{k}\left(\delta_{r}+m+1+j\right) \partial_{r}^{m+1} F(x, r) \\
& =r \sum_{i \in I(k)}[i]\left(\left\{D_{i_{0}} A_{m}^{(i)}+A_{m+1}^{\left(i^{\prime}\right)}\right\} f\right)\left(x+r p_{i_{0}}\right) \\
& \quad+r \sum_{j=1}^{k} \sum_{i \in I(j, k)}[i] \int_{x+r P_{i_{j}}}\left\{D_{i_{j}} A_{m+j}^{(i)}+A_{m+1+j}^{\left(i^{\prime}\right)}\right\} f d \nu_{i_{j}} .
\end{aligned}
$$

Dividing this equality by $r$ and using (3.13) we establish $(3.17 . m+1)$. The proof is complete.

Lemma 3.8. Let $F(x, r)$ and $\tau_{m}^{(k)}(\xi)$ be defined by (3.14) and (2.7), respectively: Then we have

$$
\prod_{j=1}^{k}(m+j)\left(\partial_{r}^{m} F\right)_{r=0}=\tau_{m}^{(k)}(\partial) f \quad(m=0,1,2, \ldots) .
$$

Proof. The formula (3.17.m) in Lemma 3.7 is rewritten as

$$
\begin{aligned}
\prod_{j=1}^{k}\left(\delta_{r}+m+j\right) \partial_{r}^{m} F= & \sum_{i \in I(k)}[i]\left(A_{m}^{(i)} f\right)\left(x+r p_{i_{0}}\right) \\
& +\sum_{j=1}^{k} r^{j} \sum_{i \in I(j, k)}[i] \int_{P_{i_{j}}}\left(A_{m+j}^{(i)} f\right)(x+r y) d v_{i_{j}}(y) .
\end{aligned}
$$

As $r \rightarrow 0$ the left-hand side tends to $\prod_{j=1}^{k}(m+j)\left(\partial_{r}^{m} F\right)_{r=0}$ and the second term in the right-hand side vanishes. Hence we obtain

$$
\begin{aligned}
\prod_{j=1}^{k}(m+j)\left(\partial_{r}^{m} F\right)_{r=0} & =\sum_{i \in I(k)}[i] A_{m}^{(i)} f \\
& =\sum_{i \in I(k)}[i] h_{m}^{(k+1)}\left(\left\langle p_{i_{0}}, \partial\right\rangle, \ldots,\left\langle p_{i_{k}}, \partial\right\rangle\right) f \\
& =\tau_{m}^{(k)}(\partial) f .
\end{aligned}
$$

Now we are in a position to establish Theorem 3.3.

Proof of Theorem 3.3. We differentiate (3.14) $m$-times with respect to $r$. Differentiations under the integral sign yield

$$
\left(\partial_{r}^{m} F\right)(x, r)=\sum_{|\alpha|=m} \frac{m !}{\alpha !} \int_{P(k)} y^{\alpha}\left(\partial^{\alpha} f\right)(x+r y) d v_{k}(y) .
$$


Putting $r=0$ we obtain

$$
\left(\partial_{r}^{m} F\right)_{r=0}=\sum_{|\alpha|=m} \frac{m !}{\alpha !} u_{\alpha} \partial^{\alpha} f
$$

where the coefficient $u_{\alpha}$ is the $\alpha$-moment of the measure $v_{k}=|P(k)| \mu$ :

$$
u_{\alpha}=\int_{P(k)} y^{\alpha} d v_{k}(y)=|P(k)| s_{\alpha} .
$$

Comparing (3.2)-(3.3) with (3.18)-(3.19) we establish the theorem.

Proof of Theorem 2.1. Theorem 2.1 is an immediate consequence of Theorems 3.1 and 3.3.

Proof of Theorem 2.2. Given any $i_{n-1} \in I_{n-1}$, let $V_{i_{n-1}}^{ \pm}$be the (closed) $n$-dimensional half-spaces having $H_{i_{n-1}}$ as their boundary, $p$ an interior point of $P_{i_{n-1}}$ in $H_{i_{n-1}}$, and $U$ a sufficiently small open neighborhood of $p$ in $\mathbb{R}^{n}$. Then for each $i_{n} \in I_{n}$ such that $i_{n-1} \prec i_{n}, P_{i_{n}} \cap U$ is contained in just one of the half-spaces $V_{i_{n-1}}^{ \pm}$. We denote it by $V_{i_{n-1}}^{\varepsilon_{i_{n}}}$. For any $i_{n}, i_{n}^{\prime} \in I_{n}$ such that $i_{n-1}<i_{n}, i_{n}^{\prime}$, we cannot have $\varepsilon_{i_{n}} \neq \varepsilon_{i_{n}^{\prime}}$; otherwise, $P_{i_{n}} \cup P_{i_{n}^{\prime}}$ would be an $n$-dimensional cell of $P$, and hence $P_{i_{n-1}}$ could not be an $(n-1)$-dimensional face of $P$ (contradiction). But $\varepsilon_{i_{n}}=\varepsilon_{i_{n}^{\prime}}$ implies $P_{i_{n}}=P_{i_{n}^{\prime}}$, i.e., $i_{n}=i_{n}^{\prime}$. Hence there exists precisely one $i_{n} \in I_{n}$ such that $i_{n-1} \prec i_{n}$. In particular, there exists a one-to-one correspondence between the index sets $I(n-1)$ and $I(n)$. Now we recall that $p_{i_{n}}=0$ for any $i_{n} \in I_{n}$. Hence for $k=n,(2.7)$ is rewritten as

$$
\begin{aligned}
\tau_{m}^{(n)}(\xi) & =\sum_{i \in I(n)}[i] h_{m}^{(n+1)}\left(\left\langle p_{i_{0}}, \xi\right\rangle,\left\langle p_{i_{1}}, \xi\right\rangle, \ldots,\left\langle p_{i_{n-1}}, \xi\right\rangle, 0\right) \\
& =\sum_{i \in I(n-1)} c_{i_{n-1}}[i] h_{m}^{(n)}\left(\left\langle p_{i_{0}}, \xi\right\rangle,\left\langle p_{i_{1}}, \xi\right\rangle, \ldots,\left\langle p_{i_{n-1}}, \xi\right\rangle\right) .
\end{aligned}
$$

We remark that $c_{i_{n-1}}=\left[i_{n-1}: i_{n}\right]$ is nonzero for at least one $i_{n-1} \in I_{n-1}$. This follows from Lemma 4.1(3) in Section 4. So if $c_{i_{n-1}}$ is independent of $i_{n-1} \in I_{n-1}$ then this common number $c$ does not vanish. Now (2.7) for $k=n-1$ and (3.20) imply that $\tau_{m}^{(n)}(\xi)=c \tau_{m}^{(n-1)}(\xi)$ for every $m$. Hence it follows from Theorem 2.1 that $\mathcal{H}_{P(n-1)}(\Omega)=$ $\mathcal{H}_{P(n)}(\Omega)$. The proof is complete.

Proposition 3.9. Let $\mu$ be as in Theorem 3.1. Assume that $\mathcal{H}_{\mu}(\Omega)$ is finite dimensional for an open subset $\Omega$ of $\mathbb{R}^{n}$.

(1) The restriction map $\mathcal{H}_{\mu}\left(\mathbb{R}^{n}\right) \rightarrow \mathcal{H}_{\mu}(\Omega)$ is an isomorphism.

(2) Any function $f \in \mathcal{H}_{\mu}\left(\mathbb{R}^{n}\right)$ satisfies the global $\mu$-mean value property, i.e., $f$ satisfies (1.1) for every $x \in \mathbb{R}^{n}$ and $r>0$.

Proof. Assertion (1): Theorem 3.1 implies that any $f \in \mathcal{H}_{\mu}\left(\mathbb{R}^{n}\right)$ is real-analytic in $\mathbb{R}^{n}$. Hence the injectivity of the restriction map $\mathcal{H}_{\mu}\left(\mathbb{R}^{n}\right) \rightarrow \mathcal{H}_{\mu}(\Omega)$ follows from the unicity theorem for real-analytic functions. We proceed to the surjectivity. Theorem 3.2 
implies that any $f \in \mathcal{H}_{\mu}(\Omega)$ is a polynomial. In particular, $f$ extends to an entire function in $\mathbb{C}^{n}$. The unicity theorem for holomorphic functions implies that $f$ satisfies the system (3.1) in $\mathbb{C}^{n}$. Theorem 3.1 implies that $f \in \mathcal{H}_{\mu}\left(\mathbb{R}^{n}\right)$. Hence the restriction map $\mathcal{H}_{\mu}\left(\mathbb{R}^{n}\right) \rightarrow \mathcal{H}_{\mu}(\Omega)$ is surjective. Assertion (2): By Assertion $(1), \mathcal{H}_{\mu}\left(\mathbb{R}^{n}\right)$ is finite dimensional. Hence Theorem 3.2 implies that any $f \in \mathcal{H}_{\mu}\left(\mathbb{R}^{n}\right)$ is a polynomial. We expand $f(x+r y)$ as $f(x+r y)=\sum_{j=0}^{m} f_{j}(x, y) r^{j}$ where $f_{j}(x, y)$ are polynomials in $(x, y)$. The $\mu$-mean value property (1.1) states that

$$
f(x)=\sum_{j=0}^{m} r^{j} \int_{K} f_{j}(x, y) d \mu(y)
$$

holds for every $0<r<r_{x}$, where $r_{x}$ is a sufficiently small positive constant depending on $x \in \mathbb{R}^{n}$. Since the both sides of (3.21) are polynomials in $r$, it is clear that $f$ satisfies (3.21) for every $r>0$.

\section{Characteristic Variety}

We are interested in the characteristic variety of the system (2.6) of partial differential equations, i.e., the common roots of the system (2.9) of algebraic equations. In addition to those in the previous sections we use the following notations. Let $I^{*}(k)$ be the subset of $I(k)$ defined by

$$
I^{*}(k)=\{i \in I(k) ;[i] \neq 0\},
$$

where $I(k)$ is given by (2.3). Given any $i_{k} \in I_{k}$, let $I\left(i_{k}\right)$ be the index set defined by

$$
I\left(i_{k}\right)=\left\{i=\left(i_{0}, \ldots, i_{k-1}, i_{k}\right) ; i_{j} \in I_{j}, i_{0} \prec \cdots \prec i_{k-1} \prec i_{k}\right\} .
$$

We remark that (4.2) is different from (2.3) since the index $i_{k}$ is now fixed; $I(k)$ is the union of $I\left(i_{k}\right)$ over all $i_{k} \in I_{k}$. Moreover, we set

$$
I^{*}\left(i_{k}\right)=I^{*}(k) \cap I\left(i_{k}\right) .
$$

Finally, let $H_{i_{k}}^{*}$ be the $k$-dimensional linear subspace of $\mathbb{R}^{n}$ parallel to the affine subspace $H_{i_{k}}$.

\section{Lemma 4.1.}

(1) For anyi $\in I\left(i_{k}\right)$, i belongsto $I^{*}\left(i_{k}\right)$ if and only if the $(k+1)$-points $p_{i_{0}}, p_{i_{1}}, \ldots, p_{i_{k}}$ are in a general position in the affine space $H_{i_{k}}$.

(2) $\sum_{i \in I^{*}\left(i_{k}\right)}[i]=k !\left|P_{i_{k}}\right|$ for any $i_{k} \in I_{k}$.

(3) $\sum_{i \in I^{*}(k)}[i]=k !|P(k)|$ for any $k=0, \ldots, n$.

Proof. For any $i=\left(i_{0}, \ldots, i_{t}, \ldots, i_{k}\right) \in I(k)$, the definition (2.1) of the vectors $p_{i_{j}}$ implies

$$
p_{i_{t}}-p_{i_{k}}=\sum_{j=t}^{k-1}\left[i_{j}: i_{j+1}\right] \mathbf{n}_{i_{j} i_{j+1}} \quad(t=0, \ldots, k-1) .
$$


The vectors $\left(\mathbf{n}_{i_{0} i_{1}}, \ldots, \mathbf{n}_{i_{k-1} i_{k}}\right)$ form an orthonormal basis of $H_{i_{k}}^{*}$. Let $\Delta_{i}$ be the $k$ dimensional simplex in $H_{i_{k}}$ defined as the convex hull of the points $p_{i_{0}}, p_{i_{1}}, \ldots, p_{i_{k}}$. Then (4.4) implies that $[i]=\left[i_{0}: i_{1}\right]\left[i_{1}: i_{2}\right] \cdots\left[i_{k-1}: i_{k}\right]$ counts $k !$-times the signed volume of the simplex $\Delta_{i}$. Three assertions of the lemma easily follow from this fact. Assertion (1): For any $i \in I\left(i_{k}\right),[i] \neq 0$ if and only if $\Delta_{i}$ has a nonzero volume. This is equivalent to the condition that $p_{0}, \ldots, p_{k}$ are in a general position in $H_{i_{k}}$. Assertion (2): The volume of $P_{i_{k}}$ equals the sum of the signed volumes of $\Delta_{i}$ for $i \in I\left(i_{k}\right)$. Clearly the sum can be taken only over $i \in I^{*}\left(i_{k}\right)$. This establishes Assertion (2). Assertion (3): Summing up the formula in Assertion (2) over $i_{k} \in I_{k}$, we obtain Assertion (3) immediately.

Lemma 4.2. For any $\xi=\left(\xi_{0}, \ldots, \xi_{k}\right) \in \mathbb{C}^{k+1}$, the following formula holds as an identity among holomorphic functions in a neighborhood of $z=0$ :

$$
\prod_{j=0}^{k}\left(1-\xi_{j} z\right)^{-1}=\sum_{m=0}^{\infty} h_{m}^{(k+1)}\left(\xi_{0}, \ldots, \xi_{k}\right) z^{m}
$$

Proof. If $\xi \neq 0$, then we put $R=\min \left|\xi_{j}\right|^{-1}$, where the minimum is taken over all $j=0, \ldots, k$ such that $\xi_{j} \neq 0$. If $\xi=0$, then we put $R=\infty$. For $|z|<R$, we have

$$
\begin{aligned}
\prod_{j=0}^{k}\left(1-\xi_{j} z\right)^{-1} & =\prod_{j=0}^{k} \sum_{m_{j}=0}^{\infty}\left(\xi_{j} z\right)^{m_{j}} \\
& =\sum_{m_{0}=0}^{\infty} \cdots \sum_{m_{k}=0}^{\infty} \xi_{0}^{m_{0}} \cdots \xi_{k}^{m_{k}} z^{m_{0}+\cdots+m_{k}} \\
& =\sum_{m=0}^{\infty}\left\{\sum_{m_{0}+\cdots+m_{k}=m} \xi_{0}^{m_{0}} \cdots \xi_{k}^{m_{k}}\right\} z^{m} \\
& =\sum_{m=0}^{\infty} h_{m}^{(k+1)}\left(\xi_{0}, \ldots, \xi_{k}\right) z^{m} .
\end{aligned}
$$

Lemma 4.3. For any $\xi \in \mathbb{C}^{n}$, the following formula holds as an identity among holomorphic functions in a neighborhood of $z=0$ :

$$
\sum_{i \in I^{*}(k)}[i] \prod_{j=0}^{k}\left(1-\left\langle p_{i_{j}}, \xi\right\rangle z\right)^{-1}=k !|P(k)|+\sum_{m=1}^{\infty} \tau_{m}^{(k)}(\xi) z^{m}
$$

Proof. Lemma 4.1(3) and Lemma 4.2 imply

$$
\sum_{i \in I(k)}[i] \prod_{j=0}^{k}\left(1-\left\langle p_{i_{j}}, \xi\right) z\right)^{-1}=\sum_{i \in I(k)}[i] \sum_{m=0}^{\infty} h_{m}^{(k+1)}\left(\left\langle p_{i_{0}}, \xi\right\rangle, \ldots,\left\langle p_{i_{k}}, \xi\right\rangle\right) z^{m}
$$




$$
\begin{aligned}
& =\sum_{m=0}^{\infty} z^{m} \sum_{i \in I(k)}[i] h_{m}^{(k+1)}\left(\left\langle p_{i_{0}}, \xi\right\rangle, \ldots,\left\langle p_{i_{k}}, \xi\right\rangle\right) \\
& =k !|P(k)|+\sum_{m=1}^{\infty} \tau_{m}^{(k)}(\xi) z^{m} .
\end{aligned}
$$

Clearly the sum in the left-hand side can be taken only over $i \in I^{*}(k)$. This establishes the lemma.

Lemma 4.4. For any $i_{k} \in I_{k}$, let $\theta_{i_{k}}: \mathbb{C}^{n} \rightarrow\{0,1\}$ be the function defined by

$$
\theta_{i_{k}}(\xi)= \begin{cases}1 & \left(\langle v, \xi\rangle=0 \text { for all } v \in H_{i_{k}}^{*} \oplus \mathbb{R} p_{i_{k}}\right), \\ 0 & \text { (otherwise) }\end{cases}
$$

If $\xi \in \mathbb{C}^{n}$ is a common root of the system (2.9) of algebraic equations, then

$$
\sum_{i_{k} \in I_{k}} \theta_{i_{k}}(\xi)\left|P_{i_{k}}\right|=|P(k)|
$$

Proof. Since $\xi \in \mathbb{C}^{n}$ is a common root of the system (2.9), Lemma 4.3 implies that

$$
\sum_{i \in I^{*}(k)}[i] \prod_{j=0}^{k}\left(1-\left\langle p_{i_{j}}, \xi\right\rangle z\right)^{-1}=k !|P(k)|
$$

holds in a neighborhood of $z=0$. Since both sides are rational in $z$ this equality holds as an identity among rational functions in $z$. Hence, we can let $z$ tend to $\infty$ in (4.9). We have

$$
\lim _{z \rightarrow \infty} \prod_{j=0}^{k}\left(1-\left\langle p_{i_{j}}, \xi\right\rangle z\right)^{-1}=\hat{\theta}_{i}(\xi) \quad\left(i \in I^{*}(k)\right)
$$

where $\hat{\theta}_{i}(\xi)$ is given by

$$
\hat{\theta}_{i}(\xi)= \begin{cases}1 & \left(\left\langle p_{i_{j}}, \xi\right\rangle=0 \text { for all } j=0, \ldots, k\right) \\ 0 & \text { (otherwise) }\end{cases}
$$

For any $i \in I^{*}\left(i_{k}\right)$ Lemma $4.1(1)$ implies that the $(k+1)$-points $p_{i_{0}}, \ldots, p_{i_{k}}$ are in a general position in $H_{i_{k}}$. Hence the vectors $p_{i_{0}}, \ldots, p_{i_{k}}$ span the linear subspace $H_{i_{k}}^{*} \oplus p_{i_{k}} \mathbb{R}$. Hence (4.7) and (4.11) imply that for any $i \in I^{*}(k)$,

$$
\hat{\theta}_{i}(\xi)=\theta_{i_{k}}(\xi) \quad \text { if } \quad i \in I^{*}\left(i_{k}\right)
$$

Applying (4.10) and (4.12) to (4.9) we obtain

$$
\begin{aligned}
k !|P(k)| & =\sum_{i \in I^{*}(k)} \hat{\theta}_{i}(\xi)[i]=\sum_{i_{k} \in I_{k}} \sum_{i \in I^{*}\left(i_{k}\right)} \theta_{i_{k}}(\xi)[i] \\
& =\sum_{i_{k} \in I_{k}} \theta_{i_{k}}(\xi) \sum_{i \in I^{*}\left(i_{k}\right)}[i]=k ! \sum_{i_{k} \in I_{k}} \theta_{i_{k}}(\xi)\left|P_{i_{k}}\right|,
\end{aligned}
$$

where Lemma 4.1(2) is used to derive the last equality. The proof is complete. 
Lemma 4.5. Let $\xi \in \mathbb{C}^{n}$ be any common root of the system (2.9) of algebraic equations. We write $\xi=\xi^{\prime}+\sqrt{-1} \xi^{\prime \prime}$, where $\xi^{\prime}$ and $\xi^{\prime \prime}$ are real vectors in $\mathbb{R}^{n}$. Then $\xi^{\prime}$ and $\xi^{\prime \prime}$ are orthogonal to the subspace $H_{i_{k}}^{*} \oplus \mathbb{R} p_{i_{k}}$ for any $i_{k} \in I_{k}$.

Proof. The identity $\sum_{i_{k} \in I_{k}}\left|P_{i_{k}}\right|=|P(k)|$ and (4.8) yields

$$
\sum_{i_{k} \in I_{k}}\left\{1-\theta_{i_{k}}(\xi)\right\}\left|P_{i_{k}}\right|=0
$$

Since $\theta_{i_{k}}(\xi)=0$ or 1 and $\left|P_{i_{k}}\right|>0$ for any $i_{k} \in I_{k}$, we must have $\theta_{i_{k}}(\xi)=1$ for any $i \in I_{k}$. Now (4.7) implies that $\langle v, \xi\rangle=0$ for all $v \in H_{i_{k}}^{*} \oplus \mathbb{R} p_{i_{k}}$. Hence the real and imaginary parts of $\xi$ are orthogonal to the space $H_{i_{k}}^{*} \oplus \mathbb{R} p_{i_{k}}$ for any $i_{k} \in I_{k}$. The proof is complete.

Lemma 4.6. For any $j=1,2, \ldots, n-1$ and $i_{j+1} \in I_{j+1}$ we have

$$
H_{i_{j+1}}^{*}=\sum_{i_{j}<i_{j+1}} H_{i_{j}}^{*}
$$

where the right-hand side is the sum of linear spaces.

Proof. For any $i_{j} \in I_{j}$ such that $i_{j} \prec i_{j+1}, P_{i_{j}}$ is a $j$-dimensional face of the $(j+1)$ dimensional polytope $P_{i_{j+1}}$. Now we recall that $H_{i_{j}}$ (resp. $\left.H_{i_{j+1}}\right)$ is the $j$ - $(\operatorname{resp} .(j+1)$-) dimensional affine subspace containing $P_{i_{j}}$ (resp. $P_{i_{j+1}}$ ) and $H_{i_{j}}^{*}$ (resp. $H_{i_{j+1}}^{*}$ ) is the $j$ (resp. $(j+1)$-) dimensional linear subspace parallel to $H_{i_{j}}$ (resp. $H_{i_{j+1}}$ ). Hence $H_{i_{j}}^{*}$ is a linear subspace of $H_{i_{j+1}}^{*}$ of codimension one. In order for $P_{i_{j+1}}$ to be a polytope there exist at least two indices $i_{j}, i_{j}^{\prime} \in I_{j}$ such that $i_{j}, i_{j}^{\prime} \prec i_{j+1}$ and $H_{i_{j}}^{*} \neq H_{i_{j}^{\prime}}^{*}$. Since $H_{i_{j}}^{*}$ and $H_{i_{j}^{\prime}}^{*}$ are of dimension $j \geq 1$ and of codimension one in $H_{i_{j+1}}^{*}$, we obtain $H_{i_{j}}^{*}+H_{i_{j}^{\prime}}^{*}=H_{i_{j+1}}^{*}$. This establish the lemma.

Now we are in a position to establish Theorems 2.3 and 1.1 .

Proof of Theorem 2.3. Let $\xi \in \mathbb{C}^{n}$ be any common root of the system (2.9). As in Lemma 4.5 , we write $\xi=\xi^{\prime}+\sqrt{-1} \xi^{\prime \prime}$. Lemma 4.5 implies that $\xi^{\prime}$ and $\xi^{\prime \prime}$ are orthogonal to $H_{i_{k}}^{*} \oplus \mathbb{R} p_{i_{k}}$ for any $i_{k} \in I_{k}$. We show that $\xi^{\prime}$ and $\xi^{\prime \prime}$ are orthogonal to $H_{i_{k+1}}^{*}$ for any $i_{k+1} \in I_{k+1}$. First we consider the case $k=0$. For any $i_{1} \in I_{1}$ there exist two indices $i_{0}, i_{0}^{\prime} \in I_{0}$ such that $i_{0}, i_{0}^{\prime} \prec i_{1}$ and the vector $p_{i_{0}}-p_{i_{0}^{\prime}}$ spans $H_{i_{1}}^{*}$. Hence $\xi^{\prime}$ and $\xi^{\prime \prime}$ are orthogonal to $H_{i_{1}}^{*}$ for any $i_{1} \in I_{1}$. In case $k \geq 1$, we apply Lemma 4 .6 for $j=k$ to show that $\xi^{\prime}$ and $\xi^{\prime \prime}$ are orthogonal to $H_{i_{k+1}}$ for any $i_{k+1} \in I_{k+1}$. Using Lemma 4.6 we repeat the same argument for $j=k+1, \ldots, n-1$. Then we eventually conclude that $\xi^{\prime}$ and $\xi^{\prime \prime}$ are orthogonal to $H_{i_{n}}^{*}=\mathbb{R}^{n}$ (for any $i_{n} \in I_{n}$ ). Hence we obtain $\xi^{\prime}=\xi^{\prime \prime}=0$, i.e., $\xi=0$. The proof is complete.

Proof of Theorem 1.1. Assertion (1) follows immediately from Theorems 2.3 and 3.2. Assertion (2) follows from the fact that the polynomials $\tau_{m}^{(k)}(\xi)$ in (2.7) are homogeneous. 
Indeed, let $f$ be any element in $\mathcal{H}_{P(k)}(\Omega)$ and write $f=\sum_{j=0}^{N} f_{j}$ for some natural number $N$, where $f_{j}$ is a homogeneous polynomial of degree $j$. Let $E=x_{1} \partial_{1}+\cdots+x_{n} \partial_{n}$ be the Euler operator. Since $\tau_{m}^{(k)}(\xi)$ is homogeneous of degree $m$, we have $\left[E, \tau_{m}^{(k)}(\partial)\right]=$ $m \tau_{m}^{(k)}(\partial)$. Hence the Euler operator $E$ keeps the solution space of (2.6) invariant. Hence Theorem 2.1 implies

$$
E^{s} f=\sum_{j=0}^{N} j^{s} f_{j} \in \mathcal{H}_{P(k)}(\Omega) \quad(s=0,1, \ldots, N) .
$$

Now the Vandermonde determinant formula implies $f_{j} \in \mathcal{H}_{P(k)}(\Omega)$ for any $j=$ $0,1, \ldots, N$. Assertion (2) is established. Assertion (3) follows easily from Theorem 2.1: If $f \in \mathcal{H}_{P(k)}(\Omega)$, then Theorem 2.1 implies that $f$ satisfies the system (2.6) of partial differential equations. Since (2.6) is a system with constant coefficients, for any $L \in \mathbb{C}[\partial]$, $L f$ also satisfies the system (2.6). Hence Theorem 2.1 again implies that $L f \in \mathcal{H}_{P(k)}(\Omega)$. The theorem is established

\section{Polytopes with Symmetry}

We show that if $P(k)$ admits ample symmetry, then any function in $\mathcal{H}_{P(k)}(\Omega)$ is harmonic in the usual sense.

Lemma 5.1. Let $G \subset O(n)$ be a group of symmetries of $P(k)$. Assume that, for any nonzero vector $v \in \mathbb{R}^{n}$, the vectors belonging to the orbit $G v$ span the entire Euclidean space $\mathbb{R}^{n}$. Then $\tau_{1}^{(k)}(\xi)=0$ and $\tau_{2}^{(k)}(\xi)=\lambda\langle\xi, \xi\rangle$ for some constant $\lambda \in \mathbb{R}$.

Proof. Since $G \subset O(n)$ is a group of symmetries of $P(k)$, the linear form $\tau_{1}^{(k)}(\xi)$ and the quadratic form $\tau_{2}^{(k)}(\xi)$ are $G$-invariant. There exists a nonzero vector $v \in \mathbb{R}^{n}$ such that $\tau_{1}^{(k)}(v)=0$. Hence $\tau_{1}^{(k)}(g v)=0$ for every $g \in G$. By assumption the vectors $g v(g \in G)$ span the entire Euclidean space $\mathbb{R}^{n}$. It follows that $\tau_{1}^{(k)}(\xi)=0$ for all $\xi \in \mathbb{R}^{n}$. Hence we obtain $\tau_{1}^{(k)}(\xi)=0$. Let $Q=\left(q_{s t}\right)$ be the symmetric matrix associated with the quadratic form $\tau_{2}^{(k)}(\xi)$. Since $\tau_{2}^{(k)}(\xi)=\sum_{s, t=1}^{n} q_{s t} \xi_{s} \xi_{t}$, it suffices to show that $Q$ is a scalar matrix. Let $\lambda$ be an eigenvalue of $Q$, and $v$ the corresponding eigenvector. The $G$-invariance of $\tau_{2}^{(k)}(\xi)$ means that the matrix $Q$ commutes with every element $g \in G$. Hence all $g v$ $(g \in G)$ are eigenvectors of $Q$ corresponding to the eigenvalue $\lambda$. Since these vectors span $\mathbb{R}^{n}$, the entire Euclidean space $\mathbb{R}^{n}$ is the eigenspace of $Q$ corresponding to the eigenvalue $\lambda$. Hence $Q$ is the scalar matrix $\lambda I$. The proof is complete.

Lemma 5.2. The constant $\lambda=\lambda_{P(k)}$ in Lemma 5.1 is explicitly given by

$$
\lambda_{P(k)}=\frac{1}{n} \sum_{i \in I(k)}[i] \sum_{j=0}^{k}(j+1)\left|p_{i_{j}}\right|^{2} .
$$

This constant is positive. 
Proof. Let $\Delta$ be the Laplacian with respect to $\xi=\left(\xi_{1}, \ldots, \xi_{n}\right)$. We use the formula $\Delta(\langle v, \xi\rangle\langle w, \xi\rangle)=2\langle v, w\rangle$ for two vectors $v, w$. Lemma 5.1 implies

$$
\tau_{2}^{(k)}(\xi)=\sum_{i \in I(k)}[i] \sum_{t=0}^{k} \sum_{s=0}^{\xi}\left\langle p_{i_{s}}, \xi\right\rangle\left\langle p_{i_{t}}, \xi\right\rangle=\lambda \sum_{j=1}^{n} \xi_{j}^{2} .
$$

Applying the Laplacian $\Delta$ to this we obtain

$$
\sum_{i \in I(k)}[i] \sum_{t=0}^{k} \sum_{s=0}^{t}\left\langle p_{i_{s}}, p_{i_{i}}\right\rangle=n \lambda
$$

For $s=0,1, \ldots, t$, the vectors $\left(\mathbf{n}_{i_{s} i_{s+1}}, \ldots, \mathbf{n}_{i_{t-1} i_{t}}, p_{i_{t}}\right)$ form an orthogonal system. Hence (4.4) yields

$$
\left\langle p_{i_{s}}, p_{i_{i}}\right\rangle=\left|p_{i_{1}}\right|^{2} \quad(s=0,1, \ldots, t) .
$$

Substituting (5.3) into (5.2) we establish (5.1). We show that the constant $\lambda$ is positive. Theorem 3.3 and Lemma 5.1 yield

$$
\sigma_{2}(\xi)=\frac{\lambda\langle\xi, \xi\rangle}{|P(k)| \prod_{j=1}^{k}(j+2)} .
$$

On the other hand. (3.2) and (3.3) imply

$$
\sigma_{2}(\xi)=\int_{P(k)}\langle\xi, y\rangle^{2} d \mu(y)
$$

(See also (1.12) in [37].) If $\sigma_{2}(\xi)=0$ for some $\xi \in \mathbb{R}^{n}$, then (5.5) implies that $\langle\xi, y\rangle=0$ for any $y \in P(k)$. Since $P(k)$ is the $k$-skeleton of a polytope in $\mathbb{R}^{n}$, the vectors $y \in P(k)$ span the entire Euclidean space $\mathbb{R}^{n}$. Hence we have $\xi=0$. So the real quadratic form $\sigma_{2}(\xi)$ is positive definite. Therefore (5.4) implies $\lambda>0$.

Proof of Theorem 2.4. Lemmas 5.1 and 5.2 imply $\tau_{2}^{(k)}(\partial)=\lambda_{P(k)} \Delta$, where $\lambda_{P(k)}$ is a positive constant and $\Delta$ is the Laplacian in $\mathbb{R}^{n}$. Hence Theorem 2.4 is an immediate consequence of Theorem 2.1 .

Proof of Theorem 2.6. Let $G$ be a cyclic group of rotations of order $N$ and assume that $G$ is a symmetry group of $P(k)$. It is well known that the homogeneous polynomials $1, z^{m}, \bar{z}^{m}(m=1,2, \ldots)$ give a basis of the harmonic polynomials in two variables. Since $N \geq 3$, for any nonzero vector $v \in \mathbb{R}^{2}$, the vectors belonging to the orbit $G v$ span the entire Euclidean plane $\mathbb{R}^{n}$. It follows from Theorem 2.4 that $\mathcal{H}_{P}(m) \subset \mathbb{C} z^{m} \oplus \mathbb{C} z^{m}$ for $m \geq 1$. For $m=1, \ldots, N-1$, we have

$$
\int_{P(k)} z^{m} d v_{k}(z)=\sum_{j=0}^{N-1} \int_{\zeta^{j} F} z^{m} d v_{k}(z)=\sum_{j=0}^{N-1} \zeta^{m j} \int_{F} z^{m} d v_{k}(z)=0,
$$


where $\zeta$ is a primitive $N$ th root of unity. This means that $z^{m}$ and $\bar{z}^{m}(m=1, \ldots, N-1)$ satisfy the $P(k)$-mean value property (1.2) at the origin. The binomial theorem

$$
z^{m}=\sum_{j=0}^{m}\left(\begin{array}{c}
m \\
j
\end{array}\right) w^{m-j}(z-w)^{j}
$$

implies that they satisfy the $P(k)$-mean value property (1.2) at any point $w \in \mathbb{C}$. Hence $1, z^{m}, \bar{z}^{m} \in \mathcal{H}_{P(k)}$ for $m=1, \ldots, N-1$. For simplicity, we put $a=a_{P(k)}$, where $a_{P(k)}$ is defined by (2.11). For $m=N$ we have

$$
\begin{aligned}
\int_{P} \operatorname{Im}\left(a z^{N}\right) d v_{k}(z) & =\frac{N}{2 \sqrt{-1}} \int_{F}\left(a z^{N}-\bar{a} \bar{z}^{N}\right) d v_{k}(z) \\
& =\frac{N}{2 \sqrt{-1}}(a \bar{a}-\bar{a} a)=0 .
\end{aligned}
$$

The binomial theorem and (5.6) implies that $\operatorname{Im}\left(a z^{N}\right) \in \mathcal{H}_{P(k)}$. By using the assumption $a \neq 0$ it is easy to see that any $f \in \mathcal{H}_{P(k)}(N)$ is a constant multiple of $\operatorname{Im}\left(a z^{N}\right)$. Finally, we show that $\mathcal{H}_{P(k)}(m)=\{0\}$ for any $m \geq N+1$. Since $\mathcal{H}_{P(k)}(m) \subset \mathbb{C} z^{m} \oplus \mathbb{C} \bar{z}^{m}$, any element $f \in \mathcal{H}_{P(k)}(m)$ is expressed as $f=\alpha z^{m}+\beta \bar{z}^{m}$ for some $\alpha, \beta \in \mathbb{C}$. Consider the differential operators:

$$
\partial_{z}=\frac{\partial}{\partial z}=\frac{1}{2}\left(\partial_{x}-\sqrt{-1} \partial_{y}\right), \quad \partial_{\bar{z}}=\frac{\partial}{\partial \bar{z}}=\frac{1}{2}\left(\partial_{x}+\sqrt{-1} \partial_{y}\right) .
$$

Since $\mathcal{H}_{P(k)}$ is a $\mathbb{C}[\partial]$-module we apply these operators repeatedly to $f$. Then we obtain

$$
\begin{aligned}
& \left(\partial_{z}\right)^{m-N} f=\alpha m(m-1) \cdots(N+1) z^{N} \in \mathcal{H}_{P(k)}, \\
& \left(\partial_{\bar{z}}\right)^{m-N} f=\beta m(m-1) \cdots(N+1) \bar{z}^{N} \in \mathcal{H}_{P(k)} .
\end{aligned}
$$

But the assumption $a \neq 0$ implies that neither $z^{N}$ nor $\bar{z}^{N}$ belongs to $\mathcal{H}_{P(k)}$. Hence we must have $\alpha=\beta=0$. The proof is complete.

\section{Examples}

The first example is regular star-polygons $P=\{N / M\}$ with center at the origin. A complete characterization of $\mathcal{H}_{P(k)}$ is given in Theorem 2.7, which we establish below.

Proof of Theorem 2.7. Any regular star-polygon $\{N / M\}$ with center at the origin admits a $\mathbb{Z}_{N}$-symmetry. To apply Theorem 2.6 we have only to show that the constant $a_{P(k)}$ in (2.11) is a nonzero real number for $k=0,1,2$. We remark that Theorem 2.2 applies to any regular star-polygon $\{N / M\}$. So the area problem $(k=2)$ and the edge problem $(k=1)$ have the same solutions. Accordingly, we have only to consider the cases $k=0,1$. We set

$$
z_{j}=\exp \left(\frac{2 \pi \sqrt{-1} j}{N}\right), \quad w_{j}=w z_{j} \quad(j=0,1, \ldots, N-1)
$$


where $w$ is the complex number defined by

$$
w=\frac{\cos (\pi M / N)}{\cos \{\pi(M-1) / N\}} \exp \left(\frac{\pi \sqrt{-1}}{N}\right) .
$$

The coordinate system $(x, y)$ is taken so that $P$ is symmetric with respect to the $x$-axis. In case $M \geq 2$ we may assume that the vertices of $P$ are given by $z_{0}, \ldots, z_{N-1}$ and $w_{0}, \ldots, w_{N-1}$. In case $M=1$ the vertices of $P$ are only $z_{0}, \ldots, z_{N-1}$. (The point $w_{j}$ is the midpoint of the edge $\left[z_{j}, z_{j+1}\right]$ in $P$.)

The vertex problem $(k=0)$ : In case $M=1$, we take the singleton set $\{1\}$ as a fundamental domain $F$. Then we have $a_{P(0)}=1$. In case $M \geq 2$ we take $F=\{1, w\}$. Then we have

$$
a_{P(0)}=1-\frac{\cos (\pi M / N)}{\cos \{\pi(M-1) / N\}}>0 .
$$

In any case $a_{P(0)}$ is a positive real number.

The edge problem $(k=1)$ : A fundamental domain $F$ of $P(1)$ is given by the union of two line segments $\left[1, w_{0}\right]$ and $\left[1, w_{N-1}\right]$. We compute the constant $a_{P(1)}$ in (2.11) with respect to the fundamental domain $F$. In terms of the polar coordinates $a_{P(1)}$ is computed as

$$
a_{P(1)}=\frac{2 \pi}{N}\left(\cos \frac{\pi M}{N}\right)^{N+1} \int_{0}^{1 / 2} u(\theta) \sin \pi \theta d \theta,
$$

where $u(\theta)$ is the function defined by

$$
\begin{aligned}
u(\theta)= & \frac{1}{\left[\cos \left\{\pi\left(M-\frac{1}{2}+\theta\right) / N\right\}\right]^{N+1}} \\
& -\frac{1}{\left[\cos \left\{\pi\left(M-\frac{1}{2}-\theta\right) / N\right\}\right]^{N+1}} .
\end{aligned}
$$

Since $u(\theta)$ is positive for $0<\theta \leq \frac{1}{2}$, the constant $a_{P(1)}$ is a positive real number. This establishes the theorem.

As the second example we consider another type of star-polygons. Let $N$ be any integer $\geq 3$. By definition a star-polygon $[2 N]$ is the union $P=P_{0} \cup P_{1}$ of two congruent regular convex $N$-gons $P_{0}$ and $P_{1}$ with center at the origin such that $P_{0} \neq P_{1}$. A star-polygon [2N] is said to be regular if the rotation of angle $\pi / N$ around the origin maps $P_{0}$ onto $P_{1}$; otherwise, it is said to be irregular. We denote by $[2 N]_{r}$ (resp. $[2 N]_{i}$ ) a regular (resp. an irregular) star-polygon [2N]. A regular star-polygon [ $2 N]_{r}$ admits a $\mathbb{Z}_{2 N}$-symmetry while an irregular star-polygon $[2 N]_{i}$ admits a $\mathbb{Z}_{N}$-symmetry. The case $N=3$ is illustrated by Figs. 6.1 and 6.2. We remark that Theorem 2.2 applies to any star-polygon [2N]. So we have only to consider the vertex $(k=0)$ and edge $(k=1)$ problems. We compute the constant $a_{P(k)}$ in (2.11) and apply Theorem 2.6 to establish the following: 


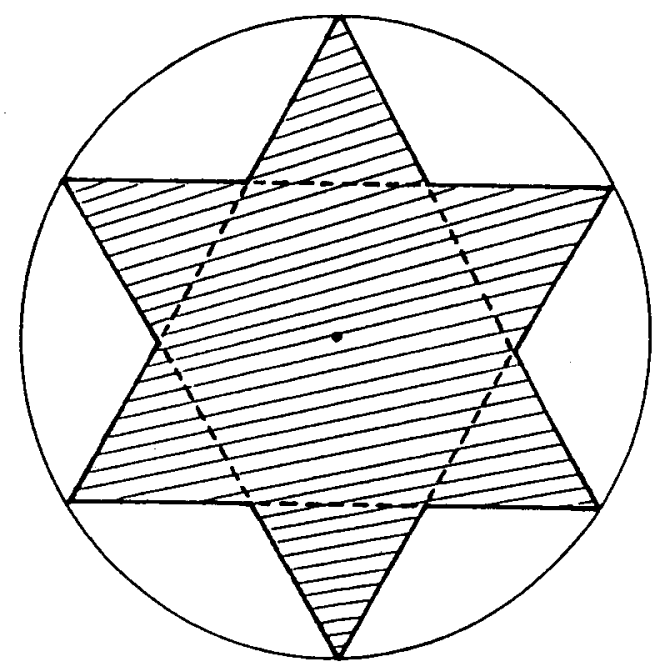

Fig. 6.1 $[6]_{r}$.

Theorem 6.1. Let $N$ be any integer $\geq 3, P$ any star-polygon $[2 N]$, and $k=0,1,2$. We take an orthonormal coordinate system $(x, y)$ of $\mathbb{R}^{2}$ such that $P$ is symmetric with respect to the $x$-axis. Then:

(1) If $P$ is a regular star-polygon $[2 N]_{r}$ then $\operatorname{dim} \mathcal{H}_{P(k)}=4 N$ and $\mathcal{H}_{P(k)}(m)$ is given by (2.12) with $N$ and $a_{P(k)}$ replaced by $2 N$ and 1 , respectively.

(2) If $P$ is an irregular star-polygon $[2 N]_{i}$ then $\operatorname{dim} \mathcal{H}_{P(k)}=2 N$ and $\mathcal{H}_{P(k)}(m)$ is given by (2.12) with $a_{P(k)}$ replaced by 1 .

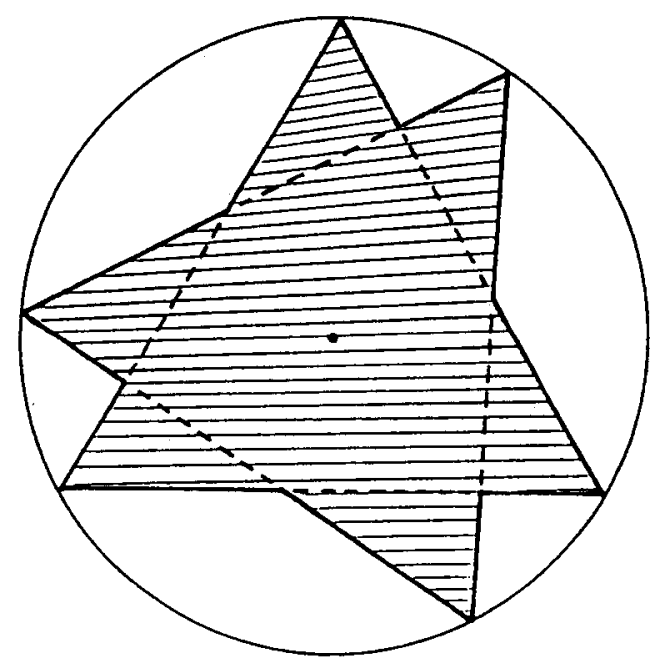

Fig. 6.2 $[6]_{i}$. 
Theorem 2.6 has many other applications of the same type, which are omitted here. Polytopes with symmetry will be discussed more thoroughly elsewhere.

Finally, we give a complete description of $\mathcal{H}_{P(k)}$ for any one-dimensional polytope. In this case the problem is quite simple but still of some interest. Any one-dimensional polytope $P$ is a finite union of closed bounded intervals

$$
P=\left[a_{1}, a_{2}\right] \cup\left[a_{3}, a_{4}\right] \cup \cdots \cup\left[a_{2 N-1}, a_{2 N}\right],
$$

in the real line $\mathbb{R}$, where $a_{1}<a_{2}<\cdots<a_{2 N}$.

Theorem 6.2. Let $P$ be any one-dimensional polytope given by (6.6). Then

$$
\begin{gathered}
\mathcal{H}_{P(0)}= \begin{cases}\mathbb{C} & \left(\sum_{j=1}^{2 N} a_{j} \neq 0\right), \\
\mathbb{C} \oplus \mathbb{C} x & \left(\sum_{j=1}^{2 N} a_{j}=0\right),\end{cases} \\
\mathcal{H}_{P(1)}= \begin{cases}\mathbb{C} & \left(\sum_{j=1}^{2 N}(-1)^{j}\left|a_{j}\right| \neq 0\right), \\
\mathbb{C} \oplus \mathbb{C} x & \left(\sum_{j=1}^{2 N}(-1)^{j}\left|a_{j}\right|=0\right) .\end{cases}
\end{gathered}
$$

Proof. The polynomials $\tau_{m}^{(k)}(\xi)$ in (2.7) are computed as

$$
\begin{aligned}
& \tau_{m}^{(0)}(\xi)=A_{m} \xi^{m}, \quad \text { where } \quad A_{m}=\sum_{j=1}^{2 N} a_{j}^{m}, \\
& \tau_{m}^{(1)}(\xi)=B_{m} \xi^{m}, \quad \text { where } \quad B_{m}=\sum_{j=1}^{2 N}(-1)^{j}\left(\operatorname{sgn} a_{j}\right) a_{j}^{m},
\end{aligned}
$$

where sgn $a_{j}$ is the signature of $a_{j}$. We have $A_{2}=\sum_{j=1}^{2 N} a_{j}^{2}>0$. So the system (2.6) is equivalent to $A_{1} \partial f=\partial^{2} f=0$. Hence Theorem 2.1 implies (6.7). Similarly, we claim $B_{2}>0$. Indeed, if $a_{2 s} \leq 0 \leq a_{2 s+1}$, then

$$
B_{2}=\sum_{j=1}^{s}\left(a_{2 j-1}^{2}-a_{2 j}^{2}\right)+\sum_{j=s+1}^{N}\left(a_{2 j}^{2}-a_{2 j-1}^{2}\right)>0,
$$

and if $a_{2 s-1} \leq 0 \leq a_{2 s}$ then

$$
B_{2}=\sum_{j=1}^{s-1}\left(a_{2 j-1}^{2}-a_{2 j}^{2}\right)+\left(a_{2 s-1}^{2}+a_{2 s}^{2}\right)+\sum_{j=s+1}^{N}\left(a_{2 j}^{2}-a_{2 j-1}^{2}\right)>0 .
$$

In any case, we have $B_{2}>0$. So the system (2.6) is equivalent to $B_{1} \partial f=\partial^{2} f=0$. Hence Theorem 2.1 implies (6.8). The proof is complete.

For $k=0,1$, the complete symmetry group $G_{P(k)}$ of $P(k)$ is given by

$$
G_{P(k)}= \begin{cases}\{1\} & \left(a_{j}+a_{2 N+1-j} \neq 0 \text { for some } j=1, \ldots, N\right), \\ \{ \pm 1\} & \left(a_{j}+a_{2 N+1-j}=0 \text { for all } j=1, \ldots, N\right),\end{cases}
$$


Hence the inclusion (2.14) is strict if and only if

$$
N \geq 2, \quad a_{j}+a_{2 N+1-j} \neq 0 \quad \text { for some } \quad j=1, \ldots, N,
$$

and

$$
\begin{cases}\sum_{j=1}^{2 N} a_{j}=0 & (k=0), \\ \sum_{j=1}^{2 N}(-1)^{j}\left|a_{j}\right|=0 & (k=1) .\end{cases}
$$

\section{Acknowledgment}

This work was done while the author was a visitor at I.R.M.A., Université Louis Pasteur. He wishes to thank Professor Raymond Gérard for his kind hospitality. Thanks are also due to the referees whose suggestions improved this paper considerably.

\section{References}

1. Aczél, J. A Mean Value Property of the Derivative of Quadratic Polynomials-Without Mean Values and Derivatives, Math. Mag. 58 (1985), 42-45.

2. Aczél, J., Haruki, H., McKiernan, M. A., and Sakovič, G. N. General and Regular Solutions of Functional Equations Characterizing Harmonic Polynomials, Aequations Math. 1 (1968), 37-53.

3. Ahern, P., Flores, M., and Rudin, W. An Invariant Volume Mean Value Property, J. Funct. Anal. 111 (1993), 380-397.

4. Armitage, D. H. The Volume Mean Value Property of Harmonic Functions, Complex Variables Theory Appl. 13 (1990), 185-193.

5. Armitage, D. H., and Nelson, C. S. A Harmonic Quadrature Formula Characterizing Open Strips, Math. Proc. Cambridge Philos. Soc. 113 (1993), 147-151.

6. Aronszajn, N. On Coercive Integro-differential Quadratic Forms, Studies in Eigenvalue Problems, Technical Report 14 (1955), 94-106.

7. Bailey, D. F. A Mean Value Property of Cubic Polynomials-Without Mean Values, Math. Mag. 65 (1992), 123-124.

8. Beckenbach, E. F., and Reade, M. O. Mean Values and Harmonic Polynomials, Trans. Amer. Math. Soc. 53 (1943), 230-238.

9. Beckenbach, E. F., and Reade, M. O. Regular Solids and Harmonic Polynomials, Duke Math. J. 12 (1945), 629-644.

10. Benyamini, Y., and Weit, Y. Functions Satisfying the Mean Value Property in the Limit, J. Analyse Math. 52 (1989), 167-198.

11. Berenstein, C. A., and Taylor, B. A. The "Three Square" Theorem for Continuous Functions, Arch. Rational Anal. Mech. 63 (1977), 253-259.

12. Berenstein, C. A., and Taylor, B. A. A New Look at Interpolation Theory of Entire Functions of One Variable, Adv. in Math. 33 (1979), 109-143.

13. Berenstein, C. A., and Taylor, B. A. Interpolation Problems in $\mathbb{C}^{n}$ with Applications to Harmonic Analysis, J. Analyse Math. 38 (1980), 188-254.

14. Björk, J.-E. Rings of Differential Operators, North-Holland, Amsterdam, 1979.

15. Bose, A. K. Functions Satisfying a Weighted Avarage Property, Trans. Amer. Math. Soc. 118 (1965), $472-487$

16. Bose, A. K. A Mean Value Property of Elliptic Equations with Constant Coefficients, Proc. Amer. Math. Soc. 18 (1967), 995-996.

17. Brödel, W. Funktionen mit Gaussicher Mittelwerteigenschaft für Konvexe Kurven und Bereiche, Deutsche Math. 4 (1939), 3-15. 
18. Bruna, J., and Detraz, J. A Converse of the Volume Mean Value Property for Invariantly Harmonic Functions, Proc. Amer. Math. Soc. 122 (1994), 1029-1034.

19. Bullen, P. S., and Sarkhel, D. N. On Darboux and Mean Value Property, Canad. Math. Bull. 30 (1987), 223-230.

20. Coxeter, H. S. M. Regular Polytopes, 3rd edn., Dover, New York, 1973.

21. Dotson, W. G., Jr. A Note on Complex Polynomials Having Roll's Property and the Mean Value Property for Derivatives, Math. Mag. 41 (1968), 140-144.

22. Epstein, B. On the Mean Value Property of Harmonic Functions, Proc. Amer. Math. Soc. 13 (1962), 830.

23. Epstein, B., and Schiffer, M. M. On the Mean Value Property of Harmonic Functions, J. Analyse Math. 14 (1965), 109-111.

24. Evans, M. J., and Fu, S. S. Mean Value Properties for Symmetrically Differentiable Functions, Real Anal. Exchange 17 (1991/92), 657-667.

25. Fenton, P. C. Functions Having the Restricted Mean Value Property, J. London Math. Soc. (2) 14 (1976), 451-458.

26. Fenton, P. C. On the Restricted Mean Value Property, Proc. Amer. Math. Soc. 100 (1987), 477-481.

27. Flatto, L. Functions with a Mean Value Property, J. Math. Mech. 10 (1961), 11-18.

28. Flatto, L. Functions with a Mean Value Property II, Amer. J. Math. 85 (1963), 248-270.

29. Flatto, L. On Polynomials Characterized by a Certain Mean Value Property, Proc. Amer. Math. Soc. 17 (1966), 598-601.

30. Flatto, L. Basic Sets of Invariants for Finite Refiection Groups, Bull. Amer. Math. Soc. 74 (1968), 730-734.

31. Flatto, L. Invariants of Finite Reflection Groups and Mean Value Problems II, Amer. J. Math. 92 (1970), $552-561$.

32. Flatto, L., and Jacobson, D. Functions Satisfying the Mean Value Property for Product Measures, Amer. J. Math. 97 (1975), 1049-1059.

33. Flatto, L., and Jacobson, D. Functions Satisfying a Discrete Mean Value Property, Aequations Math. 22 (1981), 173-193.

34. Flatto, L., and Wiener, M. M. Invariants of Finite Reflection Groups and Mean Value Problems, Amer. J. Math. 91 (1969), 591-598.

35. Flatto, L., and Wiener, M. M. Regular Polytopes and Harmonic Polynomials, Canad. J. Math. 22 (1970), 7-21.

36. Friedman, A. Mean Values and Polyharmonic Polynomials, Michigan Math. J. 4 (1957), 67-74.

37. Friedman, A., and Littman, W. Functions Satisfying the Mean Value Property, Trans. Amer. Math. Soc. 102 (1962), 167-180.

38. Friedman, A., and Littman, W. Bodies for Which Harmonic Functions Satisfy the Mean Value Property, Trans. Amer. Math. Soc. 102 (1962), 147-166.

39. Fuglede, B. On the Mean Value Property of Finely Harmonic and Finely Hyperharmonic Functions, Aequations Math. 39 (1990), 198-203.

40. Fulton, W., and Harris, J. Representation Theory, A First Course, Graduate Texts in Mathematics, No. 129. Springer-Verlag, Heidelberg, 1991.

41. Garsia, A. M. A Note on the Mean Value Property, Trans. Amer. Math. Soc. 102 (1962), 181-186.

42. Garsia, A. M., and Rodemich, E. On Functions Satisfying the Mean Value Property with Respect to a Product Measure, Proc. Amer. Math. Soc. 17 (1966), 592-594.

43. Goldstein, M., and Haussmann, W. On the Mean Value Property of Harmonic Functions and Best Harmonic $L^{1}$-Approximation, Trans. Amer. Math. Soc. 305 (1988), 505-515.

44. Goldstein, M., and Haussmann, W. On the Inverse Mean Value Property of Harmonic Functions on Strips, Bull. London Math. Soc. 24 (1992), 559-564.

45. Goldstein, M., and Ow, W. H. On the Mean Value Property of Harmonic Functions, Proc. Amer. Math. Soc. 29 (1971), 341-344.

46. Gong, X. Z. Functions with the Restricted Mean Value Property (in Chinese), Acta Sci. Natur. Univ. Amoien. 27 (1988), 611-615.

47. Haeuslein, G. K. On the Algebraic Independence of Symmetric Functions, Proc. Amer. Math. Soc. 25 (1970), 179-182.

48. Hansen, W., and Nadirashvili, N. Mean Values and Harmonic Functions, Math. Ann. 297 (1993), 157-170

49. Hansen, W., and Netuka, I. Inverse Mean Value Property for Harmonic Functions, Math. Ann. 297 (1993), 147-156. 
50. Hansen, W., and Netuka, I. Volume Densities with the Mean Value Property for Harmonic Functions, Proc. Amer. Math. Soc. 123 1995, 135-140.

51. Haruki, H. On an Integral Mean Value Theorem in Analytic Function Theory, J. London Math. Soc. 43 (1968), 551-552.

52. Haruki, H. On a Definite Integral Mean Value Problem on a Regular Polygon (in Japanese), Sûgaku 22 (1970), 131-134.

53. Haruki, H. On a Relation between the "Square" Functional Equation and the "Square" Mean Value Property, Canad. Math. Bull. 14 (1971), 161-165.

54. Haruki, S. On Two Functional Equations Connected with a Mean Value Property of Polynomials, Aequations Math. 6 (1971), 275-277.

55. Haruki, S. Four Different Unknown Functions Satisfying the Triangle Mean Value Property for Harmonic Polynomials, Ann. Polon. Math. 33 (1976/77), 219-221.

56. Haruki, S. On the Theorem of S. Kakutani-M. Nagumo and J. L. Walsh for the Mean Value Property of Harmonic and Complex Polynomials, Pacific J. Math. 94 (1981), 113-123.

57. Haruki, S. On the Mean Value Property of Harmonic and Complex Polynomials, Proc. Japan Acad. Ser. A, Math. Sci. 57 (1981), 216-218.

58. Haruki, S. Four Different Unknown Functions Satisfying the Triangle Mean Value Property for Harmonic Polynomials II, Ann. Polon. Math. 44 (1984), 239-243.

59. Iwasaki, K. Platonic Solids and the Mean Value Property, The University of Tokyo Preprint Series, 95-49, 1995.

60. Kakutani, S., and Nagumo, M. On the Functional Equation $\sum_{\nu=0}^{n-1} f\left(z+e^{2 v \pi i / \pi} \xi\right)=n f(z)$ (in Japanese), Zenk. Sugaku Zanwa. 66 (1935), 10-12.

61. Kounchev, O. I. Mean Value Property for Biharmonic Functions Characterizing the Annulus, C. R. Acad. Bulgare Sci. 42 (1989), 33-35.

62. Kuczma, M. On the Quasiarithmetric Mean in a Mean Value Property and the Associated Functional Equation, Aequations Math. 41 (1991), 33-54.

63. Kuran, U. On the Mean Value Property of Harmonic Functions, Bull. London Math. Soc. 4(1972), 31 1-312.

64. MacDonald, I. Symmetric Functions and Hall Polynomials, Oxford University Press, London, 1979.

65. McKiernan, M. A. Boundedness on a Set of Positive Measures and the Mean Value Property Characterizes Polynomials on a Space $V^{n}$, Aequations Math. 4 (1970), 31-36.

66. Muldowney, J. S. The Converse of Polya's Mean Value Theorem, SIAM J. Math. Anal. 18 (1987), 13171322.

67. Picardello, M. A., and Woess, W. A Converse to the Mean Value Property on Homogeneous Trees, Trans. Amer. Math. Soc. 311 (1989), 209-225.

68. Reade, M. O. Harmonic Polynomials, Duke Math. J. 16 (1949), 627-631.

69. Reade, M. O. Remarks on a Paper by A. Friedman, Michigan Math. J. 4 (1957), 75-76.

70. Sablik, M. A Remark on a Mean Value Property, C. R. Math. Rep. Acad. Sci. Canada 14 (1992), 207-212.

71. Sitaram, A., and Willis, G. A. $L^{p}$-Functions Satisfying the Mean Value Property on Homogeneous Spaces, J. Austral. Math. Soc. Ser. A 56 (1994), 384-390.

72. Smyrnelis, E. P. Une Propriété de Moyenne des Fonctions Biharmoniques, Bull. Sci. Math. 2e Serie 109 (1985), 103-111.

73. Stanford, D. P. Functions Satisfying a Mean Value Property at their Zeros, Amer. Math. Monthly 80 (1973), 665-667.

74. Steinberg, R. Differential Equations Invariant under Finite Reflection Groups, Trans. Amer. Math. Soc. 112 (1964), 392-400.

75. Walsh, J. L. A Mean Value Theorem for Polynomials and Harmonic Polynomials, Bull. Amer. Math. Soc. 42 (1936), 923-930.

76. Weit, Y. On a Generalized Asymptotic Mean Value Property, Aequations Math. 41 (1991), 242-247.

77. Yger, A. Fonctions Définies dans le Plan et Vérifiant Certaines Propriétés de Moyenne, Ann. Inst. Fourier 31 (1981), 115-146

78. Zalcman, L. Mean VaJues and Differential Equations, Israel J. Math. 14 (1973), 339-352.

79. Zalcman, L. Offbeat Integral Geometry, Amer. Math. Monthly 87 (1980), 161-175.

Received January 10, 1995, and in revised form December 18, 1995, and May 2, 1996. 